This is the final peer-reviewed accepted manuscript of:

Romani, L., Montagner, F.: Algebraic-Trigonometric Pythagorean-Hodograph space curves. Adv Comput Math 45, 75-98 (2019)

The final published version is available online at:

https://doi.org/10.1007/s10444-018-9606-8

Rights / License:

The terms and conditions for the reuse of this version of the manuscript are specified in the publishing policy. For all terms of use and more information see the publisher's website.

This item was downloaded from IRIS Università di Bologna (https://cris.unibo.it/)

When citing, please refer to the published version. 


\title{
Algebraic-Trigonometric Pythagorean-Hodograph space curves
}

\author{
Lucia Romani · Francesca Montagner
}

Received: date / Accepted: date

\begin{abstract}
We introduce a new class of Pythagorean-Hodograph (PH) space curves - called Algebraic-Trigonometric Pythagorean-Hodograph (ATPH) space curves - that are defined over a six-dimensional space mixing algebraic and trigonometric polynomials. After providing a general definition for this new class of curves, their quaternion representation is introduced and the fundamental properties are discussed. Then, as previously done with their quintic polynomial counterpart, a constructive approach to solve the first-order Hermite interpolation problem in $\mathbb{R}^{3}$ is provided. Comparisons with the polynomial case are illustrated to point out the greater flexibility of ATPH curves with respect to polynomial PH curves.
\end{abstract}

Keywords Space curve · Pythagorean-Hodograph · Algebraic-Trigonometric Bézier basis · Arc length · First-order Hermite Interpolation

Mathematics Subject Classification (2000) 65D17 65 D05 $\cdot 41$ A30 $\cdot 41$ A05

\section{Introduction}

The goal of this paper is to expand the boundary of Pythagorean-Hodograph $(\mathrm{PH})$ curve theory into the realm of non-polynomial curves, in order to show that the benefits of polynomial PH curves over generic polynomial curves can be extended also to curves defined over more complicated function spaces. A first attempt in this direction has been already made in [8] and [13], where the so-called Pythagorean-Hodograph cycloidal curves and Algebraic-Trigonometric Pythagorean-Hodograph (ATPH) curves were proposed as an extension of planar PH cubics and quintics, respectively. In this paper we investigate, for the first time, the existence of spatial Pythagorean-Hodograph curves defined over

L. Romani

Dipartimento di Matematica e Applicazioni, Università di Milano-Bicocca, via R. Cozzi 55, 20125 Milano, Italy (E-mail: lucia.romani@unimib.it)

F. Montagner

via S. Martino 93, 22066 Mariano Comense (CO), Italy (E-mail: f.montagner92@gmail.com) 
a six-dimensional space mixing algebraic and trigonometric polynomials. These curves are shown to be the non-polynomial analogue of the spatial PythagoreanHodograph curves defined over the six-dimensional space of quintic algebraic polynomials [6], and are thought to be a 3-dimensional extension of the planar Algebraic-Trigonometric Pythagorean-Hodograph (ATPH) curves proposed in [13]. Indeed, the idea behind our proposal is to succeed in getting a subclass of the general class of spatial Algebraic-Trigonometric curves which boasts the same advantage that the subclass of spatial polynomial PH curves possesses with respect to general spatial polynomial curves. Precisely, since spatial polynomial $\mathrm{PH}$ curves are characterized by the fact that the Euclidean norm of their hodograph (i.e. their parametric speed) is a polynomial, the subclass of spatial ATPH curves is developed in such a way that the Euclidean norm of their hodograph is a trigonometric polynomial. As a consequence, the arc length has a closed-form representation which coincides with a polynomial function in the case of $\mathrm{PH}$ curves and with a mixed algebraic-trigonometric function in the case of ATPH curves. Thus, in both cases, the arc-length can be computed explicitly without numerical quadrature. Moreover, as already shown in [13] for the planar case, when the $\mathrm{PH}$ property is extended from parametric polynomial curves to parametric curves defined over a mixed algebraic-trigonometric space, greater flexibility is achieved. In fact, an additional free parameter is introduced, which turns out to be advantageous to augment the ductility of the important class of $\mathrm{PH}$ curves in free-form design applications [12]. The same free parameter is also inherited by spatial ATPH curves and it can be used to arbitrarily modify their total arc length, to minimize their elastic bending energy, or to obtain aesthetically more pleasing curves by improving curvature and torsion distribution. In this regard we will focus our attention on the benefits offered by this shape parameter when solving a first-order Hermite interpolation problem (which constituted one of the major strands of research on PH curves over the last years [7]). For this purpose, we first revisit several important publications, such as [2-4], dealing with a general constructive approach for obtaining the two-parameter family of solutions of an analogous problem by spatial polynomial PH curves. Then, we dwell on the main differences characterizing its generalization to our non-polynomial context.

The remainder of this article is specifically organized as follows. In Section 2 we recall known results dealing with Algebraic-Trigonometric Bézier curves. Section 3 is dedicated to the definition and construction of ATPH space curves generalizing spatial PH quintics. After deriving their quaternion form, we prove that these curves have the property that the Euclidean norm of their hodograph is a trigonometric function while their arc length is a mixed algebraic-trigonometric function. In Section 4 the class of spatial ATPH curves is employed to solve the first-order Hermite interpolation problem and in Section 5 an algorithm to reconstruct from an identified spatial ATPH curve its quaternion pre-image is presented. Conclusions are drawn in Section 6.

\section{Background}

In the last decades, a great interest in the design of curves in spaces mixing algebraic, trigonometric and hyperbolic functions has arisen (see, e.g., [1,9-11]). 
Among these spaces, the only ones that turn out to be of interest in curve design are the ones that admit a normalized B-basis (see [1] for the definition of normalized B-basis and its key properties). In the following we focus our attention on a six-dimensional space mixing algebraic and trigonometric polynomials, and recall the explicit form of its normalized B-basis. Analogously to the Bernstein-Bézier basis for the space of quintic algebraic polynomials, this normalized B-basis allows us to define Algebraic-Trigonometric Bézier curves through a control polygon and evaluate them through a stable and efficient de Casteljau-type algorithm.

\subsection{Basic results on Algebraic-Trigonometric Bézier curves}

Referring to [13] for further details, in the following definitions we summarize the expressions of the normalized B-bases of two pure trigonometric spaces of our interest and of the mixed algebraic-trigonometric space used in Section 3 to define the new class of spatial Pythagorean-Hodograph curves.

Definition 1 The normalized B-bases for the pure trigonometric spaces $\tilde{U}_{2}=<$ $1, \sin (t), \cos (t)>$ and $\tilde{U}_{4}=<1, \sin (t), \cos (t), \sin (2 t), \cos (2 t)>$ are respectively defined by the set of functions $\tilde{B}_{i}^{2}:[0, \alpha] \rightarrow \mathbb{R}, i=0,1,2$ given by

$$
\begin{aligned}
\tilde{B}_{0}^{2}(t) & =\frac{\cos (\alpha-t)-1}{\cos (\alpha)-1}, \\
\tilde{B}_{1}^{2}(t) & =\frac{\cos (\alpha)-\cos (t)-\cos (\alpha-t)+1}{\cos (\alpha)-1}, \quad \text { where } 0<\alpha<\pi, \\
\tilde{B}_{2}^{2}(t) & =\frac{\cos (t)-1}{\cos (\alpha)-1},
\end{aligned}
$$

and $\tilde{B}_{i}^{4}:[0, \alpha] \rightarrow \mathbb{R}, i=0, \ldots, 4$ having the expressions

$$
\begin{aligned}
\tilde{B}_{0}^{4}(t) & =\frac{(\cos (\alpha-t)-1)^{2}}{(\cos (\alpha)-1)^{2}}, \\
\tilde{B}_{1}^{4}(t) & =\frac{2(\cos (\alpha-t)-1)(\cos (\alpha)-\cos (t)-\cos (\alpha-t)+1)}{(\cos (\alpha)-1)^{2}}, \\
\tilde{B}_{2}^{4}(t) & =\frac{2(\cos (\alpha-t)-1)(\cos (t)-1)+(\cos (\alpha)-\cos (t)-\cos (\alpha-t)+1)^{2}}{(\cos (\alpha)-1)^{2}}, \quad \text { where } \quad 0<\alpha<\frac{2}{3} \pi . \\
\tilde{B}_{3}^{4}(t) & =\frac{2(\cos (t)-1)(\cos (\alpha)-\cos (t)-\cos (\alpha-t)+1)}{(\cos (\alpha)-1)^{2}}, \\
\tilde{B}_{4}^{4}(t) & =\frac{(\cos (t)-1)^{2}}{(\cos (\alpha)-1)^{2}},
\end{aligned}
$$

Definition 2 Let $0<\alpha<\frac{2}{3} \pi$ and $\Omega=[0, \alpha]$. The normalized B-basis for the mixed algebraic-trigonometric space $U_{5}=\langle 1, t, \sin (t), \cos (t), \sin (2 t), \cos (2 t)\rangle$ is defined by the set of functions $B_{i}^{5}: \Omega \rightarrow \mathbb{R}, i=0, \ldots, 5$ given by

$$
\begin{aligned}
B_{0}^{5}(t)= & \frac{2}{n_{0}}(3(\alpha-t)+\sin (\alpha-t)(\cos (\alpha-t)-4)), \\
B_{1}^{5}(t)= & \frac{4 s_{1}}{n_{0} n_{1}}\left(n_{0} \sin ^{4}\left(\frac{\alpha-t}{2}\right)-2 s_{1}^{4}(3(\alpha-t)+\sin (\alpha-t)(\cos (\alpha-t)-4))\right), \\
B_{2}^{5}(t)= & \frac{2 s_{1}}{3 n_{2}}\left(8 \sin ^{3}\left(\frac{\alpha-t}{2}\right) \sin \left(\frac{t}{2}\right)-\frac{n_{0}}{n_{1}} \sin ^{4}\left(\frac{\alpha-t}{2}\right)\right. \\
& \left.\quad+\frac{2 s_{1}^{4}}{n_{1}}(3(\alpha-t)+\sin (\alpha-t)(\cos (\alpha-t)-4))\right), \\
B_{3}^{5}(t)= & \frac{2 s_{1}}{3 n_{2}}\left(8 \sin ^{3}\left(\frac{t}{2}\right) \sin \left(\frac{\alpha-t}{2}\right)-\frac{n_{0}}{n_{1}} \sin ^{4}\left(\frac{t}{2}\right)+\frac{2 s_{1}^{4}}{n_{1}}(3 t+\sin (t)(\cos (t)-4))\right), \\
B_{4}^{5}(t)= & \frac{4 s_{1}}{n_{0} n_{1}}\left(n_{0} \sin ^{4}\left(\frac{t}{2}\right)-2 s_{1}^{4}(3 t+\sin (t)(\cos (t)-4))\right), \\
B_{5}^{5}(t)= & \frac{2}{n_{0}}(3 t+\sin (t)(\cos (t)-4)),
\end{aligned}
$$


where

$$
s_{1}:=\sin \left(\frac{\alpha}{2}\right), \quad s_{2}:=\sin (\alpha), \quad c_{1}:=\cos \left(\frac{\alpha}{2}\right), \quad c_{2}:=\cos (\alpha)
$$

and

$$
n_{0}:=6 \alpha+2 s_{2}\left(c_{2}-4\right), \quad n_{1}:=c_{1}\left(s_{2}-3 \alpha\right)+4 s_{1}, \quad n_{2}:=\left(2+c_{2}\right) \alpha-3 s_{2} .
$$

Note that, for a function $f \in \tilde{U}_{2}$, we have $f^{2} \in \tilde{U}_{4}$ and $\int f^{2} \in U_{5}$. Specifically, when $f$ is a basis function of $\tilde{U}_{2}$ the antiderivative of $f^{2}$ assumes the following form

$$
\begin{aligned}
\int\left(\tilde{B}_{0}^{2}(t)\right)^{2} d t & =-\frac{n_{0}-6 \alpha}{16 s_{1}^{4}} B_{0}^{5}(t)+\frac{3 \alpha}{8 s_{1}^{4}} \sum_{i=1}^{5} B_{i}^{5}(t), \\
\int \tilde{B}_{0}^{2}(t) \tilde{B}_{1}^{2}(t) d t & =-\frac{s_{2}\left(7+2 c_{2}\right)}{16 s_{1}^{4}}\left(B_{0}^{5}(t)+B_{1}^{5}(t)\right)+\frac{n_{3}}{16 s_{1}^{4}} \sum_{i=2}^{5} B_{i}^{5}(t), \\
\int \tilde{B}_{0}^{2}(t) \tilde{B}_{2}^{2}(t) d t & =\frac{3 s_{2}}{16 s_{1}^{4}} \sum_{i=0}^{2} B_{i}^{5}(t)+\frac{n_{4}}{16 s_{1}^{4}} \sum_{i=3}^{5} B_{i}^{5}(t), \\
\int\left(\tilde{B}_{1}^{2}(t)\right)^{2} d t & =\frac{3 s_{2}\left(c_{2}+1\right)}{8 s_{1}^{4}} \sum_{i=0}^{2} B_{i}^{5}(t)+\frac{\left(c_{2}+1\right) n_{4}}{8 s_{1}^{4}} \sum_{i=3}^{5} B_{i}^{5}(t), \\
\int \tilde{B}_{1}^{2}(t) \tilde{B}_{2}^{2}(t) d t & =-\frac{3 s_{2}}{16 s_{1}^{4}} \sum_{i=0}^{3} B_{i}^{5}(t)+\frac{n_{5}}{16 s_{1}^{4}}\left(B_{4}^{5}(t)+B_{5}^{5}(t)\right), \\
\int\left(\tilde{B}_{2}^{2}(t)\right)^{2} d t & =\frac{n_{0}}{16 s_{1}^{4}} B_{5}^{5}(t),
\end{aligned}
$$

with

$n_{3}:=3 s_{2}-6 \alpha\left(c_{2}+1\right), \quad n_{4}:=2\left(2+c_{2}\right) \alpha-3 s_{2}, \quad n_{5}:=s_{2}\left(7+2 c_{2}\right)-6 \alpha\left(c_{2}+1\right)$.

For later use, we also point out the value of the corresponding definite integrals

$$
\begin{aligned}
\int_{0}^{\alpha}\left(\tilde{B}_{0}^{2}(t)\right)^{2} d t & =\int_{0}^{\alpha}\left(\tilde{B}_{2}^{2}(t)\right)^{2} d t=\frac{n_{0}}{16 s_{1}^{4}}=: I_{0}, \\
\int_{0}^{\alpha} \tilde{B}_{0}^{2}(t) \tilde{B}_{1}^{2}(t) d t & =\int_{0}^{\alpha} \tilde{B}_{1}^{2}(t) \tilde{B}_{2}^{2}(t) d t=\frac{n_{0}-6 n_{2}}{16 s_{1}^{4}}=: I_{1}, \\
\int_{0}^{\alpha} \tilde{B}_{0}^{2}(t) \tilde{B}_{2}^{2}(t) d t & =\frac{n_{2}}{8 s_{1}^{4}}=: I_{2}, \\
\int_{0}^{\alpha}\left(\tilde{B}_{1}^{2}(t)\right)^{2} d t & =2\left(c_{2}+1\right) \int_{0}^{\alpha} \tilde{B}_{0}^{2}(t) \tilde{B}_{2}^{2}(t) d t=2\left(c_{2}+1\right) \frac{n_{2}}{8 s_{1}^{4}}=: I_{3},
\end{aligned}
$$

where $s_{1}, c_{2}$ and $n_{0}, n_{2}$ are as in (2) and (3), respectively.

Moreover, taking into account the following equalities

$$
\begin{gathered}
\left(\tilde{B}_{0}^{2}(t)\right)^{2}=\tilde{B}_{0}^{4}(t), \quad 2 \tilde{B}_{0}^{2}(t) \tilde{B}_{1}^{2}(t)=\tilde{B}_{1}^{4}(t), \quad\left(\tilde{B}_{1}^{2}(t)\right)^{2}=\frac{1+c_{2}}{2+c_{2}} \tilde{B}_{2}^{4}(t), \\
2 \tilde{B}_{0}^{2}(t) \tilde{B}_{2}^{2}(t)=\frac{1}{2+c_{2}} \tilde{B}_{2}^{4}(t), \quad 2 \tilde{B}_{1}^{2}(t) \tilde{B}_{2}^{2}(t)=\tilde{B}_{3}^{4}(t), \quad\left(\tilde{B}_{2}^{2}(t)\right)^{2}=\tilde{B}_{4}^{4}(t),
\end{gathered}
$$

for the antiderivatives of the basis functions of $\tilde{U}_{4}$ we can write

$$
\begin{aligned}
& \int \tilde{B}_{0}^{4}(t) d t=-\frac{n_{0}-6 \alpha}{16 s_{1}^{4}} B_{0}^{5}(t)+\frac{3 \alpha}{8 s_{1}^{4}} \sum_{i=1}^{5} B_{i}^{5}(t), \\
& \int \tilde{B}_{1}^{4}(t) d t=-\frac{s_{2}\left(7+2 c_{2}\right)}{8 s_{1}^{4}}\left(B_{0}^{5}(t)+B_{1}^{5}(t)\right)+\frac{n_{3}}{8 s_{1}^{4}} \sum_{i=2}^{5} B_{i}^{5}(t), \\
& \int \tilde{B}_{2}^{4}(t) d t=\frac{3 s_{2}\left(c_{2}+2\right)}{8 s_{1}^{4}} \sum_{i=0}^{2} B_{i}^{5}(t)+\frac{n_{4}\left(c_{2}+2\right)}{8 s_{1}^{4}} \sum_{i=3}^{5} B_{i}^{5}(t), \\
& \int \tilde{B}_{3}^{4}(t) d t=-\frac{3 s_{2}}{8 s_{1}^{4}} \sum_{i=0}^{3} B_{i}^{5}(t)+\frac{n_{5}}{8 s_{1}^{4}}\left(B_{4}^{5}(t)+B_{5}^{5}(t)\right) \\
& \int \tilde{B}_{4}^{4}(t) d t=\frac{n_{0}}{16 s_{1}^{4}} B_{5}^{5}(t) .
\end{aligned}
$$

We conclude this section by recalling from [13] the following definition. 
Definition 3 Given distinct control points $\mathbf{p}_{0}, \ldots, \mathbf{p}_{5} \in \mathbb{R}^{3}$, we call algebraictrigonometric Bézier curve, or shortly AT-Bézier curve, over the mixed algebraictrigonometric space $U_{5}$, the parametric curve defined by

$$
\mathbf{r}(t)=\sum_{i=0}^{5} \mathbf{p}_{i} B_{i}^{5}(t), \quad t \in[0, \alpha], \quad 0<\alpha<\frac{2}{3} \pi
$$

where $\left\{B_{i}^{5}\right\}_{i=0}^{5}$ is the normalized B-basis of $U_{5}$ defined in (1).

We also emphasize that the basis functions $B_{i}^{5}(t), i=0, \ldots, 5$ satisfy the following properties

$$
\begin{aligned}
B_{0}^{5}(0)= & B_{5}^{5}(\alpha)=1, \quad B_{i}^{5}(0)=0, i=1, \ldots, 5, \quad B_{i}^{5}(\alpha)=0, i=0, \ldots, 4, \\
& \left(B_{0}^{5}\right)^{\prime}(0)=-\left(B_{1}^{5}\right)^{\prime}(0)=-\left(B_{5}^{5}\right)^{\prime}(\alpha)=\left(B_{4}^{5}\right)^{\prime}(\alpha)=-\frac{1}{I_{0}}, \\
& \left(B_{i}^{5}\right)^{\prime}(0)=0, i=2, \ldots, 5, \quad\left(B_{i}^{5}\right)^{\prime}(\alpha)=0, i=0, \ldots, 3,
\end{aligned}
$$

which yield

$$
\mathbf{r}(0)=\mathbf{p}_{0}, \quad \mathbf{r}(\alpha)=\mathbf{p}_{5}, \quad \mathbf{r}^{\prime}(0)=\frac{1}{I_{0}}\left(\mathbf{p}_{1}-\mathbf{p}_{0}\right), \quad \mathbf{r}^{\prime}(\alpha)=\frac{1}{I_{0}}\left(\mathbf{p}_{5}-\mathbf{p}_{4}\right),
$$

with $I_{0}$ in (5). Moreover, spatial AT-Bézier curves defined over $U_{5}$ can be efficiently and stably evaluated by the de Casteljau-like algorithm proposed in [13]. These are key properties that are successively used to extend the construction of spatial Pythagorean-Hodograph curves from the space of quintic algebraic polynomials to the mixed algebraic-trigonometric space $U_{5}$.

\section{Quaternion form of Algebraic-Trigonometric Pythagorean-Hodograph space curves and their distinctive features}

The goal of this section is to generalize the definition of Pythagorean-Hodograph space curves (see [2, Chapter 21]) to Algebraic-Trigonometric Pythagorean-Hodograph space curves.

Definition 4 Let $u(t), v(t), p(t), q(t)$ be real trigonometric functions in $\tilde{U}_{2}$. Then, the spatial parametric curve $\mathbf{r}(t)=(x(t), y(t), z(t))$ whose coordinate components have first derivatives of the form

$$
\begin{aligned}
& x^{\prime}(t)=u^{2}(t)+v^{2}(t)-p^{2}(t)-q^{2}(t) \\
& y^{\prime}(t)=2[u(t) q(t)+v(t) p(t)] \\
& z^{\prime}(t)=2[v(t) q(t)-u(t) p(t)]
\end{aligned}
$$

is called an Algebraic-Trigonometric Pythagorean-Hodograph space curve.

Remark 1 Definition 4 is supported by the fact that if $u, v, p, q \in \tilde{U}_{2}$, then $x^{\prime}, y^{\prime}, z^{\prime} \in$ $\tilde{U}_{4}$ and verify the Pythagorean condition

$$
\left(x^{\prime}(t)\right)^{2}+\left(y^{\prime}(t)\right)^{2}+\left(z^{\prime}(t)\right)^{2}=(\sigma(t))^{2}
$$

with $\sigma(t)=u^{2}(t)+v^{2}(t)+p^{2}(t)+q^{2}(t)$. 
Proposition 1 If $x^{\prime}, y^{\prime}, z^{\prime}, \sigma$ are real trigonometric functions in $\tilde{U}_{4}$ with no common roots, and satisfy the Pythagorean condition

$$
\left(x^{\prime}(t)\right)^{2}+\left(y^{\prime}(t)\right)^{2}+\left(z^{\prime}(t)\right)^{2}=(\sigma(t))^{2},
$$

then they can be expressed in terms of real trigonometric functions $u, v, p, q \in \tilde{U}_{2}$ in the form

$$
\begin{aligned}
x^{\prime}(t) & =u^{2}(t)+v^{2}(t)-p^{2}(t)-q^{2}(t), \\
y^{\prime}(t) & =2[u(t) q(t)+v(t) p(t)], \\
z^{\prime}(t) & =2[v(t) q(t)-u(t) p(t)], \\
\sigma(t) & =u^{2}(t)+v^{2}(t)+p^{2}(t)+q^{2}(t) .
\end{aligned}
$$

Proof The proof is obtained by replicating the steps of the proof of $[2$, Theorem 21.1], where polynomial functions of degree 2 and 4 are replaced by trigonometric functions in $\tilde{U}_{2}$ and $\tilde{U}_{4}$, respectively.

As we will see later, it is convenient to represent the hodograph of a spatial ATPH curve

$\mathbf{r}^{\prime}(t)=\left[u^{2}(t)+v^{2}(t)-p^{2}(t)-q^{2}(t)\right] \mathbf{i}+2[u(t) q(t)+v(t) p(t)] \mathbf{j}+2[v(t) q(t)-u(t) p(t)] \mathbf{k}$,

using its quaternion form. Precisely, if after introducing the quaternion function

$$
\mathbf{A}(t)=u(t)+v(t) \mathbf{i}+p(t) \mathbf{j}+q(t) \mathbf{k}
$$

we consider its conjugate

$$
\mathbf{A}^{*}(t)=u(t)-v(t) \mathbf{i}-p(t) \mathbf{j}-q(t) \mathbf{k},
$$

we can easily obtain

$$
\mathbf{r}^{\prime}(t)=\mathbf{A}(t) \mathbf{i} \mathbf{A}^{*}(t)
$$

Proposition 2 The spatial ATPH curve can be expressed in the AT-Bézier form

$$
\mathbf{r}(t)=\sum_{i=0}^{5} \mathbf{p}_{i} B_{i}^{5}(t), \quad t \in[0, \alpha],
$$

with $3 D$ control points $\mathbf{p}_{i}, i=1, \ldots, 5$ of the form

$$
\begin{aligned}
& \mathbf{p}_{\mathbf{1}}=\mathbf{p}_{\mathbf{0}}+I_{0} \mathbf{A}_{0} \mathbf{i} \mathbf{A}_{0}^{*}, \\
& \mathbf{p}_{\mathbf{2}}=\mathbf{p}_{\mathbf{1}}+I_{1}\left(\mathbf{A}_{0} \mathbf{i} \mathbf{A}_{1}^{*}+\mathbf{A}_{1} \mathbf{i} \mathbf{A}_{0}^{*}\right) \\
& \mathbf{p}_{\mathbf{3}}=\mathbf{p}_{\mathbf{2}}+I_{2}\left(\mathbf{A}_{0} \mathbf{i} \mathbf{A}_{2}^{*}+\mathbf{A}_{2} \mathbf{i} \mathbf{A}_{0}^{*}\right)+I_{3} \mathbf{A}_{1} \mathbf{i} \mathbf{A}_{1}^{*}, \\
& \mathbf{p}_{\mathbf{4}}=\mathbf{p}_{\mathbf{3}}+I_{1}\left(\mathbf{A}_{1} \mathbf{i} \mathbf{A}_{2}^{*}+\mathbf{A}_{2} \mathbf{i} \mathbf{A}_{1}^{*}\right) \\
& \mathbf{p}_{\mathbf{5}}=\mathbf{p}_{\mathbf{4}}+I_{0} \mathbf{A}_{2} \mathbf{i} \mathbf{A}_{2}^{*},
\end{aligned}
$$

where $I_{0}, I_{1}, I_{2}, I_{3}$ are defined as in (5) and the integration constant $\mathbf{p}_{0}$ is freely chosen. 
Proof By substituting the trigonometric function $\mathbf{A}(t)=\mathbf{A}_{0} \tilde{B}_{0}^{2}(t)+\mathbf{A}_{1} \tilde{B}_{1}^{2}(t)+$ $\mathbf{A}_{2} \tilde{B}_{2}^{2}(t)$ with quaternion coefficients $\mathbf{A}_{\ell}=u_{\ell}+v_{\ell} \mathbf{i}+p_{\ell} \mathbf{j}+q_{\ell} \mathbf{k}, \ell=0,1,2$ into the hodograph form (9), we obtain

$$
\begin{aligned}
\mathbf{r}^{\prime}(t) & =\mathbf{A}_{0} \mathbf{i} \mathbf{A}_{0}^{*}\left(\tilde{B}_{0}^{2}(t)\right)^{2}+\mathbf{A}_{1} \mathbf{i} \mathbf{A}_{1}^{*}\left(\tilde{B}_{1}^{2}(t)\right)^{2}+\mathbf{A}_{2} \mathbf{i} \mathbf{A}_{2}^{*}\left(\tilde{B}_{2}^{2}(t)\right)^{2} \\
& +\left(\mathbf{A}_{0} \mathbf{i} \mathbf{A}_{1}^{*}+\mathbf{A}_{1} \mathbf{i} \mathbf{A}_{0}^{*}\right) \tilde{B}_{0}^{2}(t) \tilde{B}_{1}^{2}(t)+\left(\mathbf{A}_{1} \mathbf{i} \mathbf{A}_{2}^{*}+\mathbf{A}_{2} \mathbf{i} \mathbf{A}_{1}^{*}\right) \tilde{B}_{1}^{2}(t) \tilde{B}_{2}^{2}(t) \\
& +\left(\mathbf{A}_{0} \mathbf{i} \mathbf{A}_{2}^{*}+\mathbf{A}_{2} \mathbf{i} \mathbf{A}_{0}^{*}\right) \tilde{B}_{0}^{2}(t) \tilde{B}_{2}^{2}(t) .
\end{aligned}
$$

Since $\mathbf{r}(t)=\int \mathbf{r}^{\prime}(t) d t$, by integrating the expression in (11) exploiting the formulae in (4), we obtain the AT-Bézier form of $\mathbf{r}(t)$ with control points in (10).

Remark 2 In the well-known polynomial case, the control points of the spatial $\mathrm{PH}$ quintic have exactly the form in (10) (see, e.g., [3]), where $I_{0}=\frac{1}{5}, I_{1}=\frac{1}{10}$, $I_{2}=\frac{1}{30}, I_{3}=\frac{2}{15}$. These are indeed the values of the definite integrals in (4), obtained by setting $\alpha=1$ and replacing $\tilde{B}_{j}^{2}(t), j=0,1,2$ with the degree-2 Bernstein polynomials.

\subsection{Parametric speed}

The parametric speed of the ATPH curve $\mathbf{r}(t)$ is the rate of change $\sigma=\frac{d s}{d t}$ of its arc length $s$ with respect to the curve parameter $t$. As in the case of spatial PH polynomial curves [2, Chapters 21-22], the parametric speed of $\mathbf{r}(t)=$ $(x(t), y(t), z(t))$ is given by

$$
\sigma(t)=\left|\mathbf{r}^{\prime}(t)\right|=\sqrt{\left(x^{\prime}(t)\right)^{2}+\left(y^{\prime}(t)\right)^{2}+\left(z^{\prime}(t)\right)^{2}}=u^{2}(t)+v^{2}(t)+p^{2}(t)+q^{2}(t) .
$$

Since the square of a function in $\tilde{U}_{2}$ is a function in $\tilde{U}_{4}$, the parametric speed of a spatial ATPH curve is a trigonometric function in $\tilde{U}_{4}$. The fact that $\sigma(t)$ is a trigonometric function (rather than the square-root of a trigonometric function) in $t$ is the source of the advantageous properties of ATPH curves. To compute the explicit expression of $\sigma(t)$ in the B-basis of $\tilde{U}_{4}$ we need to compute

$$
\sigma(t)=\left|\mathbf{A}(t) \mathbf{i} \mathbf{A}^{*}(t)\right|=|\mathbf{A}(t)|^{2}=\mathbf{A}(t) \mathbf{A}^{*}(t)
$$

with

$\mathbf{A}(t)=\mathbf{A}_{0} \tilde{B}_{0}^{2}(t)+\mathbf{A}_{1} \tilde{B}_{1}^{2}(t)+\mathbf{A}_{2} \tilde{B}_{2}^{2}(t), \quad \mathbf{A}^{*}(t)=\mathbf{A}_{0}^{*} \tilde{B}_{0}^{2}(t)+\mathbf{A}_{1}^{*} \tilde{B}_{1}^{2}(t)+\mathbf{A}_{2}^{*} \tilde{B}_{2}^{2}(t)$,

and

$$
\mathbf{A}_{\ell}=u_{\ell}+v_{\ell} \mathbf{i}+p_{\ell} \mathbf{j}+q_{\ell} \mathbf{k}, \quad \mathbf{A}_{\ell}^{*}=u_{\ell}-v_{\ell} \mathbf{i}-p_{\ell} \mathbf{j}-q_{\ell} \mathbf{k}, \quad \ell=0,1,2
$$

the corresponding quaternion coefficients. There follows that

$$
\begin{aligned}
\sigma(t) & =\left|\mathbf{A}_{0}\right|^{2}\left(\tilde{B}_{0}^{2}(t)\right)^{2}+\left|\mathbf{A}_{1}\right|^{2}\left(\tilde{B}_{1}^{2}(t)\right)^{2}+\left|\mathbf{A}_{2}\right|^{2}\left(\tilde{B}_{2}^{2}(t)\right)^{2} \\
& +\left(\mathbf{A}_{1} \mathbf{A}_{0}^{*}+\mathbf{A}_{0} \mathbf{A}_{1}^{*}\right) \tilde{B}_{0}^{2}(t) \tilde{B}_{1}^{2}(t)+\left(\mathbf{A}_{1} \mathbf{A}_{2}^{*}+\mathbf{A}_{2} \mathbf{A}_{1}^{*}\right) \tilde{B}_{1}^{2}(t) \tilde{B}_{2}^{2}(t) \\
& +\left(\mathbf{A}_{2} \mathbf{A}_{0}^{*}+\mathbf{A}_{0} \mathbf{A}_{2}^{*}\right) \tilde{B}_{0}^{2}(t) \tilde{B}_{2}^{2}(t) .
\end{aligned}
$$


At this point, we exploit formulae (6) to arrive at

$$
\begin{aligned}
\sigma(t) & =\left|\mathbf{A}_{0}\right|^{2} \tilde{B}_{0}^{4}(t)+\frac{1}{2}\left(\mathbf{A}_{1} \mathbf{A}_{0}^{*}+\mathbf{A}_{0} \mathbf{A}_{1}^{*}\right) \tilde{B}_{1}^{4}(t) \\
& +\frac{1}{2+c_{2}}\left(\left(1+c_{2}\right)\left|\mathbf{A}_{1}\right|^{2}+\frac{1}{2}\left(\mathbf{A}_{2} \mathbf{A}_{0}^{*}+\mathbf{A}_{0} \mathbf{A}_{2}^{*}\right)\right) \tilde{B}_{2}^{4}(t) \\
& +\frac{1}{2}\left(\mathbf{A}_{1} \mathbf{A}_{2}^{*}+\mathbf{A}_{2} \mathbf{A}_{1}^{*}\right) \tilde{B}_{3}^{4}(t)+\left|\mathbf{A}_{2}\right|^{2} \tilde{B}_{4}^{4}(t) .
\end{aligned}
$$

To conclude

$$
\sigma(t)=\sum_{i=0}^{4} \sigma_{i} \tilde{B}_{i}^{4}(t)
$$

where

$$
\begin{aligned}
& \sigma_{0}=\left|\mathbf{A}_{0}\right|^{2}, \\
& \sigma_{1}=\frac{1}{2}\left(\mathbf{A}_{1} \mathbf{A}_{0}^{*}+\mathbf{A}_{0} \mathbf{A}_{1}^{*}\right), \\
& \sigma_{2}=\frac{1}{2+c_{2}}\left(\left(1+c_{2}\right)\left|\mathbf{A}_{1}\right|^{2}+\frac{1}{2}\left(\mathbf{A}_{2} \mathbf{A}_{0}^{*}+\mathbf{A}_{0} \mathbf{A}_{2}^{*}\right)\right), \\
& \sigma_{3}=\frac{1}{2}\left(\mathbf{A}_{1} \mathbf{A}_{2}^{*}+\mathbf{A}_{2} \mathbf{A}_{1}^{*}\right), \\
& \sigma_{4}=\left|\mathbf{A}_{2}\right|^{2} .
\end{aligned}
$$

\subsection{Cumulative and total arc length}

In this section we show that the cumulative arc length is an algebraic-trigonometric function of the curve parameter $t$, and the total arc length can be computed exactly (i.e., without numerical quadrature) by rational arithmetic on the curve coefficients. To this end, we exploit equation (13) to write the arc length function

$$
s(t)=\int \sigma(t) d t=\int \sum_{i=0}^{4} \sigma_{i} \tilde{B}_{i}^{4}(t) d t=\sum_{i=0}^{4} \sigma_{i} \int \tilde{B}_{i}^{4}(t) d t .
$$

Then, recalling formulae (7) we arrive at

$$
\begin{aligned}
s(t) & =\frac{1}{8 s_{1}^{4}}\left(s_{2}\left(4-c_{2}\right) \sigma_{0}-s_{2}\left(7+2 c_{2}\right) \sigma_{1}+3 s_{2}\left(c_{2}+2\right) \sigma_{2}-3 s_{2} \sigma_{3}\right) B_{0}^{5}(t) \\
& +\frac{1}{8 s_{1}^{4}}\left(3 \alpha \sigma_{0}-s_{2}\left(7+2 c_{2}\right) \sigma_{1}+3 s_{2}\left(c_{2}+2\right) \sigma_{2}-3 s_{2} \sigma_{3}\right) B_{1}^{5}(t) \\
& +\frac{1}{8 s_{1}^{4}}\left(3 \alpha \sigma_{0}+n_{3} \sigma_{1}+3 s_{2}\left(c_{2}+2\right) \sigma_{2}-3 s_{2} \sigma_{3}\right) B_{2}^{5}(t) \\
& +\frac{1}{8 s_{1}^{4}}\left(3 \alpha \sigma_{0}+n_{3} \sigma_{1}+n_{4}\left(c_{2}+2\right) \sigma_{2}-3 s_{2} \sigma_{3}\right) B_{3}^{5}(t) \\
& +\frac{1}{8 s_{1}^{4}}\left(3 \alpha \sigma_{0}+n_{3} \sigma_{1}+n_{4}\left(c_{2}+2\right) \sigma_{2}+n_{5} \sigma_{3}\right) B_{4}^{5}(t) \\
& +\frac{1}{8 s_{1}^{4}}\left(3 \alpha \sigma_{0}+n_{3} \sigma_{1}+n_{4}\left(c_{2}+2\right) \sigma_{2}+n_{5} \sigma_{3}+\left(3 \alpha-s_{2}\left(4-c_{2}\right)\right) \sigma_{4}\right) B_{5}^{5}(t),
\end{aligned}
$$

namely the arc length function is an algebraic-trigonometric function in $U_{5}$. There follows that the cumulative arc length of an ATPH curve is

$$
\begin{aligned}
\mathcal{S}(\xi) & =\int_{0}^{\xi} \sigma(t) d t=s(\xi)-s(0) \\
& =\frac{1}{8 s_{1}^{4}}\left(s_{2}\left(4-c_{2}\right) \sigma_{0}-s_{2}\left(7+2 c_{2}\right) \sigma_{1}+3 s_{2}\left(c_{2}+2\right) \sigma_{2}-3 s_{2} \sigma_{3}\right)\left(B_{0}^{5}(\xi)-1\right) \\
& +\frac{1}{8 s_{1}^{4}}\left(3 \alpha \sigma_{0}-s_{2}\left(7+2 c_{2}\right) \sigma_{1}+3 s_{2}\left(c_{2}+2\right) \sigma_{2}-3 s_{2} \sigma_{3}\right) B_{1}^{5}(\xi) \\
& +\frac{1}{8 s_{1}^{4}}\left(3 \alpha \sigma_{0}+n_{3} \sigma_{1}+3 s_{2}\left(c_{2}+2\right) \sigma_{2}-3 s_{2} \sigma_{3}\right) B_{2}^{5}(\xi) \\
& +\frac{1}{8 s_{1}^{4}}\left(3 \alpha \sigma_{0}+n_{3} \sigma_{1}+n_{4}\left(c_{2}+2\right) \sigma_{2}-3 s_{2} \sigma_{3}\right) B_{3}^{5}(\xi) \\
& +\frac{1}{8 s_{1}^{4}}\left(3 \alpha \sigma_{0}+n_{3} \sigma_{1}+n_{4}\left(c_{2}+2\right) \sigma_{2}+n_{5} \sigma_{3}\right) B_{4}^{5}(\xi) \\
& +\frac{1}{8 s_{1}^{4}}\left(3 \alpha \sigma_{0}+n_{3} \sigma_{1}+n_{4}\left(c_{2}+2\right) \sigma_{2}+n_{5} \sigma_{3}+\left(3 \alpha-s_{2}\left(4-c_{2}\right)\right) \sigma_{4}\right) B_{5}^{5}(\xi)
\end{aligned}
$$


and the total arc length $S$ of an ATPH curve can be written as

$$
S=\int_{0}^{\alpha} \sigma(t) d t=\mathcal{S}(\alpha)=\frac{1}{8 s_{1}^{4}}\left(\frac{n_{0}}{2}\left(\sigma_{0}+\sigma_{4}\right)+\left(n_{0}-6 n_{2}\right)\left(\sigma_{1}+\sigma_{3}\right)+2\left(c_{2}+2\right) n_{2} \sigma_{2}\right) .
$$

\section{First-order spatial Algebraic-Trigonometric Pythagorean-Hodograph Hermite interpolants}

Given arbitrary control points $\mathbf{p}_{0} \neq \mathbf{p}_{1}$ and $\mathbf{p}_{4} \neq \mathbf{p}_{5}$ of an AT-Bézier curve $\mathbf{r}(t)=\sum_{i=0}^{5} \mathbf{p}_{i} B_{i}^{5}(t), t \in[0, \alpha]$, defined over the space $U_{5}$, we look for the two remaining inner control points $\mathbf{p}_{2}$ and $\mathbf{p}_{3}$ such that all six are expressible in the form given by equations (10) for some quaternions $\mathbf{A}_{0}, \mathbf{A}_{1}, \mathbf{A}_{2}$. Since ATBézier curves built-upon the normalized B-basis $B_{i}^{5}, i=0, \ldots, 5$ satisfy (8), this problem can be obviously regarded as a first-order Hermite interpolation problem to prescribed end points $\mathbf{p}_{0}, \mathbf{p}_{5}$ and end first derivatives at these end points. Hereinafter the first derivatives at $\mathbf{p}_{0}, \mathbf{p}_{5}$ will be denoted by $\mathbf{d}_{i}, \mathbf{d}_{f}$, respectively. Following the line of reasoning in [2, Section 28.2], the following result can be proven.

Proposition 3 Let $s_{1}, c_{2}, n_{0}, n_{2}$ be as in (2)-(3), $I_{1}, I_{3}$ as in (5) and

$$
\begin{aligned}
& M:=\frac{1}{64 s_{1}^{8}}\left(\frac{\left(n_{0}-6 n_{2}\right)^{2}}{4}-\left(c_{2}+1\right) n_{0} n_{2}\right), \\
& N:=\frac{1}{32 s_{1}^{8}}\left(\frac{\left(n_{0}-6 n_{2}\right)^{2}}{8}-\left(c_{2}+1\right)\left(n_{2}\right)^{2}\right) .
\end{aligned}
$$

The ATPH curves $\mathbf{r}(t)$ solving the first-order Hermite interpolation problem

$$
\mathbf{r}(0)=\mathbf{p}_{0}, \quad \mathbf{r}^{\prime}(0)=\mathbf{d}_{i}, \quad \mathbf{r}(\alpha)=\mathbf{p}_{5}, \quad \mathbf{r}^{\prime}(\alpha)=\mathbf{d}_{f},
$$

have control points given by expressions (10) with

$$
\begin{gathered}
\mathbf{A}_{0}=\sqrt{\left|\mathbf{d}_{i}\right|} \frac{\mathbf{i}+\mathbf{w}_{i}}{\left|\mathbf{i}+\mathbf{w}_{i}\right|} \exp \left(\phi_{0} \mathbf{i}\right), \quad \mathbf{A}_{2}=\sqrt{\left|\mathbf{d}_{f}\right|} \frac{\mathbf{i}+\mathbf{w}_{f}}{\left|\mathbf{i}+\mathbf{w}_{f}\right|} \exp \left(\phi_{2} \mathbf{i}\right), \\
\mathbf{A}_{1}=-\frac{I_{1}}{I_{3}}\left(\mathbf{A}_{0}+\mathbf{A}_{2}\right)+\frac{\sqrt{|\mathbf{c}|}}{I_{3}} \frac{\mathbf{i}+\mathbf{w}_{c}}{\left|\mathbf{i}+\mathbf{w}_{c}\right|} \exp \left(\phi_{1} \mathbf{i}\right),
\end{gathered}
$$

where

$$
\mathbf{c}:=I_{3}\left(\mathbf{p}_{5}-\mathbf{p}_{0}\right)+M\left(\mathbf{d}_{i}+\mathbf{d}_{f}\right)+N\left(\mathbf{A}_{0} \mathbf{i} \mathbf{A}_{2}^{*}+\mathbf{A}_{2} \mathbf{i} \mathbf{A}_{0}^{*}\right),
$$

and

- $\left(\lambda_{i}, \mu_{i}, \nu_{i}\right),\left(\lambda_{f}, \mu_{f}, \nu_{f}\right),\left(\lambda_{c}, \mu_{c}, \nu_{c}\right)$ are the direction cosines of $\mathbf{d}_{i}, \mathbf{d}_{f}$ and $\mathbf{c}$, respectively,

- $\mathbf{w}_{i}=\lambda_{i} \mathbf{i}+\mu_{i} \mathbf{j}+\nu_{i} \mathbf{k}, \mathbf{w}_{f}=\lambda_{f} \mathbf{i}+\mu_{f} \mathbf{j}+\nu_{f} \mathbf{k}, \mathbf{w}_{c}=\lambda_{c} \mathbf{i}+\mu_{c} \mathbf{j}+\nu_{c} \mathbf{k}$ are unit vectors in the directions of $\mathbf{d}_{i}, \mathbf{d}_{f}$ and $\mathbf{c}$, respectively,

- $\phi_{0}, \phi_{1}, \phi_{2}$ are free angular variables varying in the interval $\left[-\frac{\pi}{2}, \frac{\pi}{2}\right]$. 
Proof In view of (9) and (12), interpolation of the end-derivatives yields the equations

$$
\mathbf{A}_{0} \mathbf{i} \mathbf{A}_{0}^{*}=\mathbf{d}_{i}, \quad \mathbf{A}_{2} \mathbf{i} \mathbf{A}_{2}^{*}=\mathbf{d}_{f},
$$

for $\mathbf{A}_{0}$ and $\mathbf{A}_{2}$, where $\mathbf{d}_{i}$ and $\mathbf{d}_{f}$ are known pure vectors. Moreover, interpolation of the end points $\mathbf{p}_{0}$ and $\mathbf{p}_{5}$ (with $\mathbf{p}_{0}$ the integration constant) gives the condition

$$
\begin{aligned}
\int_{0}^{\alpha} \mathbf{A}(t) \mathbf{i} \mathbf{A}^{*}(t) d t=\mathbf{p}_{5}-\mathbf{p}_{0} & =I_{0} \mathbf{A}_{0} \mathbf{i} \mathbf{A}_{0}^{*}+I_{1}\left(\mathbf{A}_{0} \mathbf{i} \mathbf{A}_{1}^{*}+\mathbf{A}_{1} \mathbf{i} \mathbf{A}_{0}^{*}\right) \\
& +I_{2}\left(\mathbf{A}_{0} \mathbf{i} \mathbf{A}_{2}^{*}+\mathbf{A}_{2} \mathbf{i} \mathbf{A}_{0}^{*}\right)+I_{3} \mathbf{A}_{1} \mathbf{i} \mathbf{A}_{1}^{*} \\
& +I_{1}\left(\mathbf{A}_{1} \mathbf{i} \mathbf{A}_{2}^{*}+\mathbf{A}_{2} \mathbf{i} \mathbf{A}_{1}^{*}\right)+I_{0} \mathbf{A}_{2} \mathbf{i} \mathbf{A}_{2}^{*} .
\end{aligned}
$$

Recalling the result in [3, Section 3.2], the quaternion equations (19) can be solved directly to obtain

$$
\begin{aligned}
\mathbf{A}_{0} & =\sqrt{\frac{\left(1+\lambda_{i}\right)}{2}\left|\mathbf{d}_{i}\right|}\left(-\sin \phi_{0}+\cos \phi_{0} \mathbf{i}+\frac{\mu_{i} \cos \phi_{0}+\nu_{i} \sin \phi_{0}}{1+\lambda_{i}} \mathbf{j}+\frac{\nu_{i} \cos \phi_{0}-\mu_{i} \sin \phi_{0}}{1+\lambda_{i}} \mathbf{k}\right) \\
& =\sqrt{\left|\mathbf{d}_{i}\right|} \frac{\mathbf{i}+\mathbf{w}_{i}}{\left|\mathbf{i}+\mathbf{w}_{i}\right|} \exp \left(\phi_{0} \mathbf{i}\right) \quad \text { with } \quad \mathbf{w}_{i}=\lambda_{i} \mathbf{i}+\mu_{i} \mathbf{j}+\nu_{i} \mathbf{k},
\end{aligned}
$$

and

$$
\begin{aligned}
\mathbf{A}_{2} & =\sqrt{\frac{\left(1+\lambda_{f}\right)}{2}\left|\mathbf{d}_{f}\right|}\left(-\sin \phi_{2}+\cos \phi_{2} \mathbf{i}+\frac{\mu_{f} \cos \phi_{2}+\nu_{f} \sin \phi_{2}}{1+\lambda_{f}} \mathbf{j}+\frac{\nu_{f} \cos \phi_{2}-\mu_{f} \sin \phi_{2}}{1+\lambda_{f}} \mathbf{k}\right) \\
& =\sqrt{\left|\mathbf{d}_{f}\right|} \frac{\mathbf{i}+\mathbf{w}_{f}}{\left|\mathbf{i}+\mathbf{w}_{f}\right|} \exp \left(\phi_{2} \mathbf{i}\right) \quad \text { with } \quad \mathbf{w}_{f}=\lambda_{f} \mathbf{i}+\mu_{f} \mathbf{j}+\nu_{f} \mathbf{k},
\end{aligned}
$$

where $\left(\lambda_{i}, \mu_{i}, \nu_{i}\right)$ and $\left(\lambda_{f}, \mu_{f}, \nu_{f}\right)$ are the direction cosines of $\mathbf{d}_{i}$ and $\mathbf{d}_{f}$, while $\phi_{0}$ and $\phi_{2}$ are free angular variables. Knowing $\mathbf{A}_{0}$ and $\mathbf{A}_{2}$, the solution of (20) for $\mathbf{A}_{1}$ may appear more difficult. However, by using (19) and making appropriate rearrangements, this equation can be written as

$$
\begin{aligned}
\left(I_{1} \mathbf{A}_{0}+I_{3} \mathbf{A}_{1}+I_{1} \mathbf{A}_{2}\right) \mathbf{i}\left(I_{1} \mathbf{A}_{0}+I_{3} \mathbf{A}_{1}+I_{1} \mathbf{A}_{2}\right)^{*} & =I_{3}\left(\mathbf{p}_{5}-\mathbf{p}_{0}\right)+M\left(\mathbf{d}_{i}+\mathbf{d}_{f}\right) \\
& +N\left(\mathbf{A}_{0} \mathbf{i} \mathbf{A}_{2}^{*}+\mathbf{A}_{2} \mathbf{i} \mathbf{A}_{0}^{*}\right),
\end{aligned}
$$

with $I_{1}, I_{3}$ as in (5) and $M, N$ as in (16). Equation (23) is of the form $\widehat{\mathbf{A}} \mathbf{i} \widehat{\mathbf{A}}^{*}=\mathbf{c}$ (exactly as (19)) with

$$
\widehat{\mathbf{A}}:=I_{1} \mathbf{A}_{0}+I_{3} \mathbf{A}_{1}+I_{1} \mathbf{A}_{2}
$$

and $\mathbf{c}$ in (18). Note that $\mathbf{c}$ is a known pure vector. In fact, $\mathbf{A}_{0} \mathbf{i} \mathbf{A}_{2}^{*}+\mathbf{A}_{2} \mathbf{i} \mathbf{A}_{0}^{*}$ is twice the vector part of $\mathbf{A}_{0} \mathbf{i} \mathbf{A}_{2}^{*}$ in light of the fact that $\mathbf{A}_{2} \mathbf{i} \mathbf{A}_{0}^{*}=\left(\mathbf{A}_{0} \mathbf{i} \mathbf{A}_{2}^{*}\right)^{*}$. Exploiting (21) and (22), we can write

$$
\mathbf{A}_{0} \mathbf{i} \mathbf{A}_{2}^{*}+\mathbf{A}_{2} \mathbf{i} \mathbf{A}_{0}^{*}=\sqrt{\left(1+\lambda_{i}\right)\left|\mathbf{d}_{i}\right|\left(1+\lambda_{f}\right)\left|\mathbf{d}_{f}\right|}\left(a_{x} \mathbf{i}+a_{y} \mathbf{j}+a_{z} \mathbf{k}\right),
$$

where

$$
\begin{aligned}
& a_{x}=\cos (\Delta \phi)-\frac{\left(\mu_{i} \mu_{f}+\nu_{i} \nu_{f}\right) \cos (\Delta \phi)+\left(\mu_{i} \nu_{f}-\mu_{f} \nu_{i}\right) \sin (\Delta \phi)}{\left(1+\lambda_{i}\right)\left(1+\lambda_{f}\right)}, \\
& a_{y}=\frac{\mu_{i} \cos (\Delta \phi)-\nu_{i} \sin (\Delta \phi)}{1+\lambda_{i}}+\frac{\mu_{f} \cos (\Delta \phi)+\nu_{f} \sin (\Delta \phi)}{1+\lambda_{f}}, \\
& a_{z}=\frac{\nu_{i} \cos (\Delta \phi)+\mu_{i} \sin (\Delta \phi)}{1+\lambda_{i}}+\frac{\nu_{f} \cos (\Delta \phi)-\mu_{f} \sin (\Delta \phi)}{1+\lambda_{f}}
\end{aligned}
$$


and

$$
\Delta \phi:=\phi_{2}-\phi_{0}
$$

Finally, writing $\mathbf{c}=c_{x} \mathbf{i}+c_{y} \mathbf{j}+c_{z} \mathbf{k}$, the solution of (23) for $\mathbf{A}_{1}$ is

$$
\begin{aligned}
\mathbf{A}_{1} & =-\frac{I_{1}}{I_{3}}\left(\mathbf{A}_{0}+\mathbf{A}_{2}\right)+\frac{\sqrt{\frac{\left(1+\lambda_{c}\right)}{2}|\mathbf{c}|}}{I_{3}}\left(-\sin \phi_{1}+\cos \phi_{1} \mathbf{i}+\frac{\mu_{c} \cos \phi_{1}+\nu_{c} \sin \phi_{1}}{1+\lambda_{c}} \mathbf{j}\right. \\
& \left.+\frac{\nu_{c} \cos \phi_{1}-\mu_{c} \sin \phi_{1}}{1+\lambda_{c}} \mathbf{k}\right) \\
& =-\frac{I_{1}}{I_{3}}\left(\mathbf{A}_{0}+\mathbf{A}_{2}\right)+\frac{\sqrt{|\mathbf{c}|}}{I_{3}} \frac{\mathbf{i}+\mathbf{w}_{c}}{\left|\mathbf{i}+\mathbf{w}_{c}\right|} \exp \left(\phi_{1} \mathbf{i}\right) \quad \text { with } \quad \mathbf{w}_{c}=\lambda_{c} \mathbf{i}+\mu_{c} \mathbf{j}+\nu_{c} \mathbf{k},
\end{aligned}
$$

where $\left(\lambda_{c}, \mu_{c}, \nu_{c}\right)$ are the direction cosines of $\mathbf{c}$, while $\phi_{1}$ is another free angular variable.

Proposition 4 The total arc length $S$ of an ATPH Hermite interpolant has the compact quaternion representation

$$
S=\frac{1}{I_{3}}\left(\widehat{\mathbf{A}} \widehat{\mathbf{A}}^{*}-M\left(\mathbf{A}_{0} \mathbf{A}_{0}^{*}+\mathbf{A}_{2} \mathbf{A}_{2}^{*}\right)-N\left(\mathbf{A}_{0} \mathbf{A}_{2}^{*}+\mathbf{A}_{2} \mathbf{A}_{0}^{*}\right)\right),
$$

with $\widehat{\mathbf{A}}$ in (24), $I_{3}$ in (5) and $M, N$ in (16).

Proof Substituting into equation (15) the values of $\sigma_{i}, i=0, \ldots, 4$ in (14) we obtain

$$
\begin{aligned}
S & =I_{0}\left(\mathbf{A}_{0} \mathbf{A}_{0}^{*}+\mathbf{A}_{2} \mathbf{A}_{2}^{*}\right)+I_{1}\left(\mathbf{A}_{1} \mathbf{A}_{0}^{*}+\mathbf{A}_{0} \mathbf{A}_{1}^{*}+\mathbf{A}_{1} \mathbf{A}_{2}^{*}+\mathbf{A}_{2} \mathbf{A}_{1}^{*}\right) \\
& +I_{2}\left(\mathbf{A}_{0} \mathbf{A}_{2}^{*}+\mathbf{A}_{2} \mathbf{A}_{0}^{*}\right)+I_{3} \mathbf{A}_{1} \mathbf{A}_{1}^{*},
\end{aligned}
$$

with $I_{0}, I_{1}, I_{2}, I_{3}$ the abbreviations in (5). Noting that $I_{0}$ and $I_{2}$ verify the identities

$$
I_{0}=\frac{\left(I_{1}\right)^{2}-M}{I_{3}}, \quad I_{2}=\frac{\left(I_{1}\right)^{2}-N}{I_{3}},
$$

for $M, N$ in (16), equation (26) can be further rewritten in the equivalent form

$$
\begin{aligned}
S=\frac{1}{I_{3}} & \left(\left(I_{1}\right)^{2} \mathbf{A}_{0} \mathbf{A}_{0}^{*}+I_{1} I_{3} \mathbf{A}_{0} \mathbf{A}_{1}^{*}+\left(I_{1}\right)^{2} \mathbf{A}_{0} \mathbf{A}_{2}^{*}+I_{1} I_{3} \mathbf{A}_{1} \mathbf{A}_{0}^{*}+\left(I_{3}\right)^{2} \mathbf{A}_{1} \mathbf{A}_{1}^{*}\right. \\
& +I_{1} I_{3} \mathbf{A}_{1} \mathbf{A}_{2}^{*}+\left(I_{1}\right)^{2} \mathbf{A}_{2} \mathbf{A}_{0}^{*}+I_{1} I_{3} \mathbf{A}_{2} \mathbf{A}_{1}^{*}+\left(I_{1}\right)^{2} \mathbf{A}_{2} \mathbf{A}_{2}^{*} \\
& \left.-M\left(\mathbf{A}_{0} \mathbf{A}_{0}^{*}+\mathbf{A}_{2} \mathbf{A}_{2}^{*}\right)-N\left(\mathbf{A}_{0} \mathbf{A}_{2}^{*}+\mathbf{A}_{2} \mathbf{A}_{0}^{*}\right)\right)
\end{aligned}
$$

which is nothing but the expanded form of (25).

Corollary 1 Assuming $\phi_{1}$ to be fixed, the total arc length $S$ of a spatial ATPH Hermite interpolant depends only on $\alpha$ and the difference $\Delta \phi=\phi_{2}-\phi_{0}$ of the two angular degrees of freedom.

Proof If $\phi_{1}$ is fixed, it is easy to see that the quaternion $\widehat{\mathbf{A}}=I_{1} \mathbf{A}_{0}+I_{3} \mathbf{A}_{1}+I_{1} \mathbf{A}_{2}$ and its conjugate depend only on $\alpha$ and $\Delta \phi$. In fact, in light of (17), $\widehat{\mathbf{A}}$ can be rewritten as $\widehat{\mathbf{A}}=\sqrt{|\mathbf{c}|} \frac{\mathbf{i}+\mathbf{w}_{c}}{\left|\mathbf{i}+\mathbf{w}_{c}\right|} \exp \left(\phi_{1} \mathbf{i}\right)$, with $\mathbf{c}$ the pure vector in (18) depending only on $\alpha$ and $\Delta \phi$. Moreover, we can replace the term $\mathbf{A}_{0} \mathbf{A}_{0}^{*}+\mathbf{A}_{2} \mathbf{A}_{2}^{*}$ by the constant $\left|\mathbf{d}_{i}\right|+\left|\mathbf{d}_{f}\right|$, and also easily verify that $\mathbf{A}_{0} \mathbf{A}_{2}^{*}+\mathbf{A}_{2} \mathbf{A}_{0}^{*}$ depends only on $\Delta \phi$. Thus, $S$ depends both on $\alpha$ and $\Delta \phi$ only. 
Remark 3 We observe that, for a given set of Hermite data, the arc lengths $S$ of $\mathrm{PH}$ Hermite interpolants lie in a relatively narrow range, while the range where the arc lengths $S$ of ATPH Hermite interpolants can vary is much larger. For example, if we consider the Hermite data $\mathbf{p}_{0}=(0,0,0), \mathbf{p}_{5}=(1,1,1), \mathbf{d}_{i}=(1,0,1)$, $\mathbf{d}_{f}=(0,1,1)$ and assume $\phi_{1}=-\frac{\pi}{2}$, the arc lengths of PH Hermite interpolants lie in $[1.777,1.825]$ while the arc lengths of ATPH Hermite interpolants lie in $[1.734,1.999]$. This lower bound of $S$ for ATPH Hermite interpolants is obtained with $\alpha=0.05$, while the upper bound is achieved when $\alpha=\frac{2}{3} \pi-0.05$.

\subsection{Independent degrees of freedom of spatial ATPH Hermite interpolants}

The three angular variables $\phi_{0}, \phi_{1}, \phi_{2}$, associated with the quaternions $\mathbf{A}_{0}, \mathbf{A}_{1}$, $\mathbf{A}_{2}$ respectively, do not identify independent degrees of freedom. In fact, the control points in (10) depend only on the products $\mathbf{A}_{r} \mathbf{i} \mathbf{A}_{s}^{*}$ which can be written as $\mathbf{A}_{r} \mathbf{i} \mathbf{A}_{s}^{*}=\mathbf{A}_{r}(0)\left(\sin \left(\phi_{s}-\phi_{r}\right)+\cos \left(\phi_{s}-\phi_{r}\right) \mathbf{i}\right) \mathbf{A}_{s}^{*}(0)$, by introducing the notation $\mathbf{A}_{r}:=\mathbf{A}_{r}\left(\phi_{r}\right)=\mathbf{A}_{r}(0)\left(\cos \phi_{r}+\sin \phi_{r} \mathbf{i}\right)$. Thus the control points of spatial ATPH Hermite interpolants indeed depend only on $\alpha$ and the difference of the angles $\phi_{0}, \phi_{1}, \phi_{2}$. Without loss of generality, we can assume $\phi_{1}$ to be fixed and, recalling [3], we can conveniently select $\phi_{1}=-\frac{\pi}{2}$. In this way, the first-order Hermite interpolation problem admits a three-parameter family of solutions since, besides $\phi_{0}$ and $\phi_{2}$, the value of $\alpha$ also identifies a degree of freedom.

Remark 4 In the quintic polynomial case, the Hermite interpolation problem was proven to yield a two-parameter family of solutions (see, e.g., [2-4]) since $\phi_{0}$ and $\phi_{2}$ are the only two independent degrees of freedom. When $\alpha=1$ is selected, the families of ATPH Hermite interpolants behave closely to the corresponding families of PH Hermite interpolants (Figure 1). In this figure, as well as in many others of this section, we refer to color lines to distinguish among multiple plots. We thus invite the reader to refer to the electronic version of the paper to make the identification of individual plots easier.

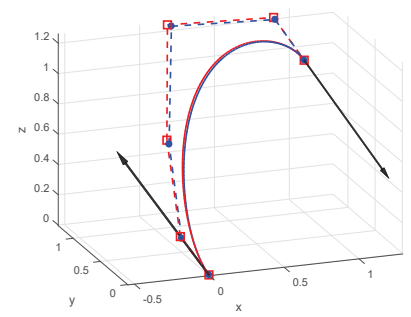

(a) $\phi_{0}=-\frac{\pi}{2}, \phi_{2}=-\frac{\pi}{2}$

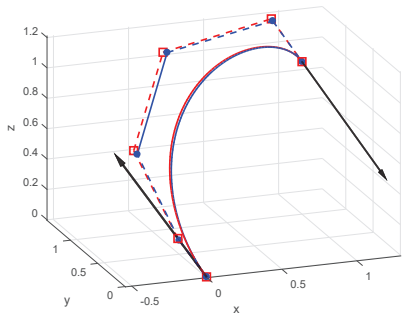

(b) $\phi_{0}=-\frac{\pi}{3}, \phi_{2}=-\frac{2 \pi}{5}$

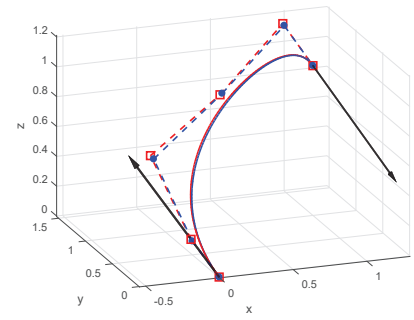

(c) $\phi_{0}=-\frac{\pi}{3}, \phi_{2}=-\frac{\pi}{5}$

Fig. 1 Comparison between ATPH (solid red) and PH (solid blue) Hermite interpolants to the data $\mathbf{p}_{0}=(0,0,0), \mathbf{p}_{5}=(1,1,1), \mathbf{d}_{i}=(-0.8,0.3,1.2), \mathbf{d}_{f}=(0.5,-1.3,-1)$, obtained by fixing $\alpha=1$ and $\phi_{1}=-\frac{\pi}{2}$, and choosing $\phi_{0}, \phi_{2}$ as specified in each subfigure. The ATPH and $\mathrm{PH}$ control polygons are delineated by a dashed red and blue polyline, respectively.

Recalling the previously introduced notation $\Delta \phi=\phi_{2}-\phi_{0}$, we can write

$$
\phi_{0}=\phi_{m}-\frac{1}{2} \Delta \phi \quad \text { and } \quad \phi_{2}=\phi_{m}+\frac{1}{2} \Delta \phi, \quad \text { for } \quad \phi_{m}:=\frac{1}{2}\left(\phi_{0}+\phi_{2}\right) .
$$


Figure 2 illustrates how the arc length of spatial ATPH curves interpolating the same Hermite data, changes in terms of $\alpha, \phi_{m}$ (a), $\Delta \phi, \phi_{m}$ (b) and $\alpha, \Delta \phi$ (c).

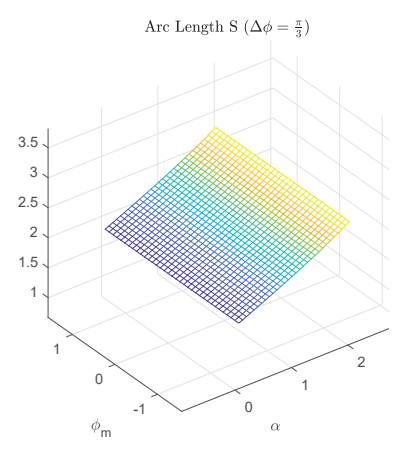

(a)

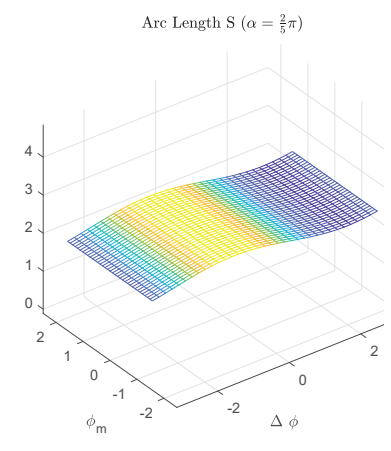

(b)

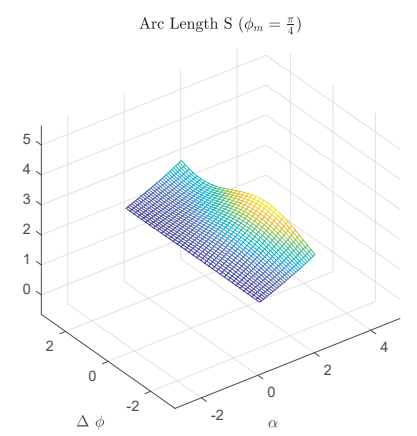

(c)

Fig. 2 Behaviour of the arc length $S$ for the families of ATPH Hermite interpolants to the data $\mathbf{p}_{0}=(0,0,0), \mathbf{p}_{5}=(1,1,1), \mathbf{d}_{i}=(-0.8,0.3,1.2), \mathbf{d}_{f}=(0.5,-1.3,-1)$, obtained by fixing $\phi_{1}=-\frac{\pi}{2}$ and assuming: $\alpha \in\left(0, \frac{2}{3} \pi\right), \phi_{m} \in\left[-\frac{\pi}{2}, \frac{\pi}{2}\right]$ (a), $\Delta \phi \in[-\pi, \pi], \phi_{m} \in\left[-\frac{\pi}{2}, \frac{\pi}{2}\right]$ (b), $\alpha \in\left(0, \frac{2}{3} \pi\right), \Delta \phi \in[-\pi, \pi]$ (c).

a) In the first situation considered in Figure 2, we assume $\Delta \phi$ constant and change $\alpha$ and $\phi_{m}$ so obtaining:

a.1) for a fixed value of $\alpha$, a family of spatial ATPH Hermite interpolants with the same arc length but varying shapes (see Figure 3);

a.2) for a fixed value of $\phi_{m}$, a family of spatial ATPH Hermite interpolants with the same shape but varying arc length (see Figure 4).

Indeed, according to Figure 2(a), we can observe that, for a fixed value of $\phi_{m}, S$ becomes larger and larger when considering increasing values of $\alpha$. Differently, for a fixed value of $\alpha, S$ does not change with $\phi_{m}$.

b) In the second situation considered in Figure 2, we assume $\alpha$ constant and change $\Delta \phi$ and $\phi_{m}$ (exactly like in the quintic polynomial case) so obtaining:

b.1) for a fixed value of $\Delta \phi$, a family of spatial ATPH Hermite interpolants with the same arc length but varying shapes (see Figure 5);

b.2) for a fixed value of $\phi_{m}$, a family of spatial ATPH Hermite interpolants with varying shapes and varying arc length (see Figure 6).

Indeed, according to Figure 2(b), we can observe that, for a fixed value of $\Delta \phi, S$ does not change with $\phi_{m}$. Moreover, monotonically increasing values of $\Delta \phi$ do not provide monotonically increasing values of $S$. Differently, for a fixed value of $\phi_{m}, S$ changes with $\Delta \phi$, but still not monotonically.

c) Finally, in the third situation considered in Figure 2, we assume $\phi_{m}$ constant and change $\alpha$ and $\Delta \phi$ so obtaining:

c.1) for a fixed value of $\alpha$, spatial ATPH Hermite interpolants with varying shapes and varying arc length (see Figure 7);

c.2) for a fixed value of $\Delta \phi$, spatial ATPH Hermite interpolants with the same shape but varying arc length (see Figure 8).

Indeed, according to Figure 2(c), we can observe that, for a fixed value of $\alpha$, $S$ changes with $\Delta \phi$, but not in a monotonic way. Differently, for a fixed value of $\Delta \phi, S$ increases in a monotonic way by considering increasing values of $\alpha$. 


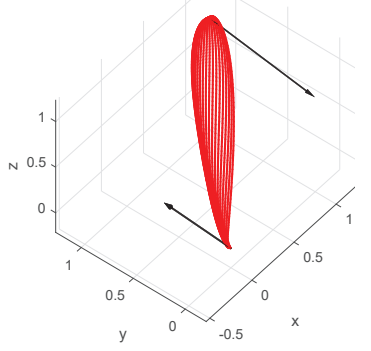

(a) $\alpha=\frac{2}{21} \pi, S=1.8634$

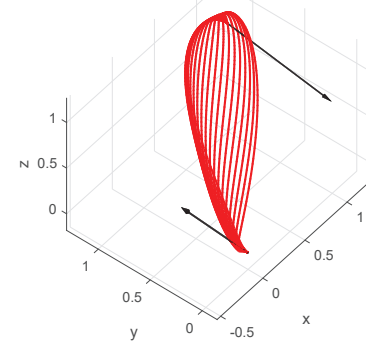

(b) $\alpha=\frac{2}{7} \pi, S=2.1331$

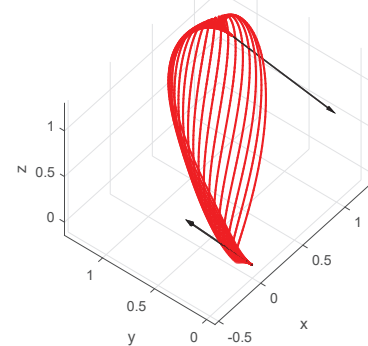

(c) $\alpha=\frac{10}{21} \pi, S=2.4253$

Fig. 3 One-parameter families of spatial ATPH Hermite interpolants to the data $\mathbf{p}_{0}=$ $(0,0,0), \mathbf{p}_{5}=(1,1,1), \mathbf{d}_{i}=(-0.8,0.3,1.2), \mathbf{d}_{f}=(0.5,-1.3,-1)$, defined by fixing $\phi_{1}=-\frac{\pi}{2}$, $\Delta \phi=\frac{\pi}{3}$ and varying only $\phi_{m}$ in $\left[-\frac{\pi}{2}, \frac{\pi}{2}\right]$.

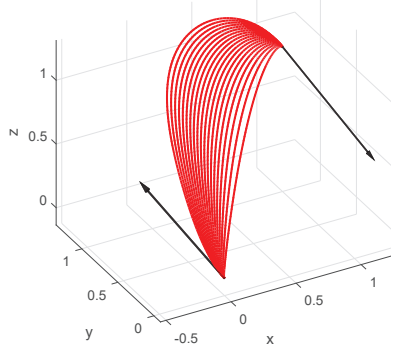

(a) $\phi_{m}=-\frac{\pi}{2}$

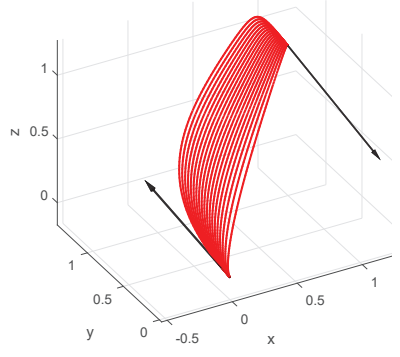

(b) $\phi_{m}=-\frac{\pi}{10}$

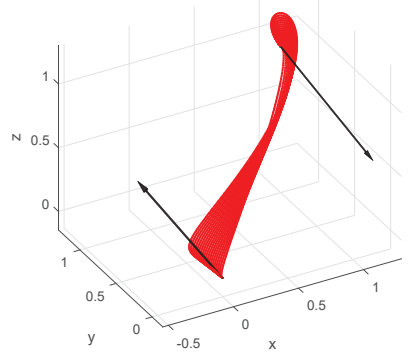

(c) $\phi_{m}=\frac{3}{10} \pi$

Fig. 4 One-parameter families of spatial ATPH Hermite interpolants to the data $\mathbf{p}_{0}=$ $(0,0,0), \mathbf{p}_{5}=(1,1,1), \mathbf{d}_{i}=(-0.8,0.3,1.2), \mathbf{d}_{f}=(0.5,-1.3,-1)$, defined by fixing $\phi_{1}=-\frac{\pi}{2}$, $\Delta \phi=\frac{\pi}{3}$ and varying only $\alpha \in\left(0, \frac{2}{3} \pi\right)$. In each subfigure, assuming $\alpha_{j}=\frac{\pi}{30} j, j=1, \ldots, 19$, curves with increasing arc length values are obtained.

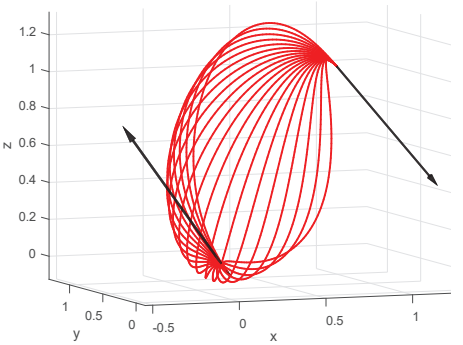

(a) $\Delta \phi=-\frac{3}{5} \pi, S=2.4778$

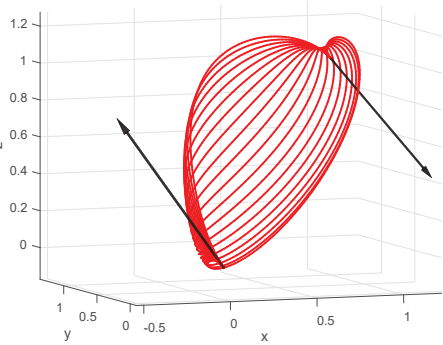

(b) $\Delta \phi=\frac{\pi}{5}, S=2.3861$

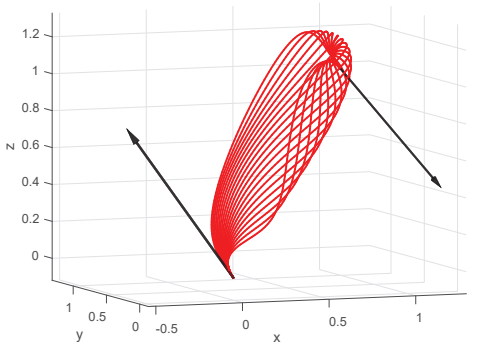

(c) $\Delta \phi=\pi, S=2.2386$

Fig. 5 One-parameter families of spatial ATPH Hermite interpolants to the data $\mathbf{p}_{0}=$ $(0,0,0), \mathbf{p}_{5}=(1,1,1), \mathbf{d}_{i}=(-0.8,0.3,1.2), \mathbf{d}_{f}=(0.5,-1.3,-1)$, defined by fixing $\phi_{1}=-\frac{\pi}{2}$, $\alpha=\frac{2 \pi}{5}$ and varying only $\phi_{m}$ in $\left[-\frac{\pi}{2}, \frac{\pi}{2}\right]$. 


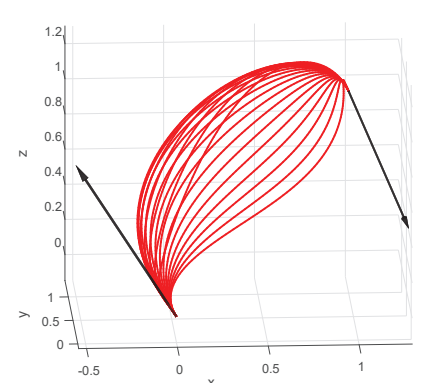

(a) $\phi_{m}=-\frac{3}{10} \pi$

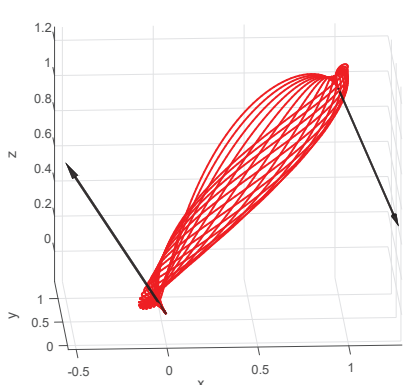

(b) $\phi_{m}=\frac{\pi}{10}$

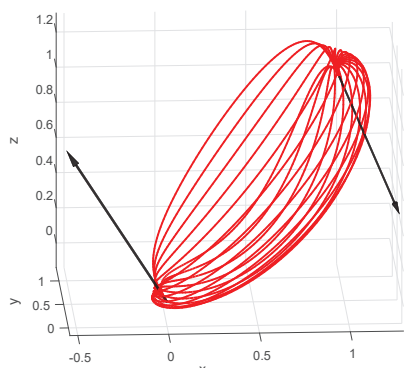

(c) $\phi_{m}=\frac{\pi}{2}$

Fig. 6 One-parameter families of spatial ATPH Hermite interpolants to the data $\mathbf{p}_{0}=$ $(0,0,0), \mathbf{p}_{5}=(1,1,1), \mathbf{d}_{i}=(-0.8,0.3,1.2), \mathbf{d}_{f}=(0.5,-1.3,-1)$, defined by fixing $\phi_{1}=-\frac{\pi}{2}$, $\alpha=\frac{2 \pi}{5}$, and varying only $\Delta \phi$ in $[-\pi, \pi]$. In each subfigure, assuming $(\Delta \phi)_{j}=\frac{2 j-21}{19} \pi$, $j=1, \ldots, 20$, curves with different arc length values are obtained.

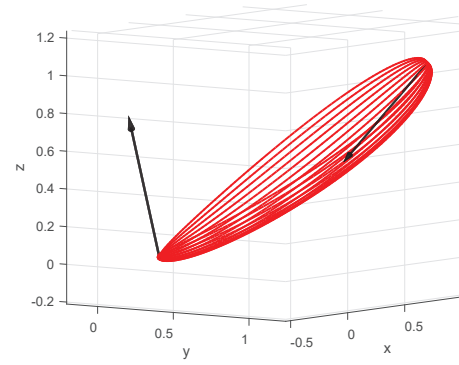

(a) $\alpha=\frac{2}{21} \pi$

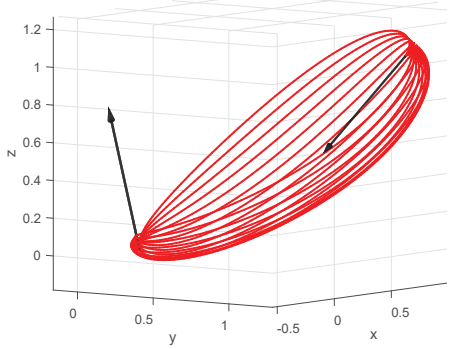

(b) $\alpha=\frac{2}{7} \pi$

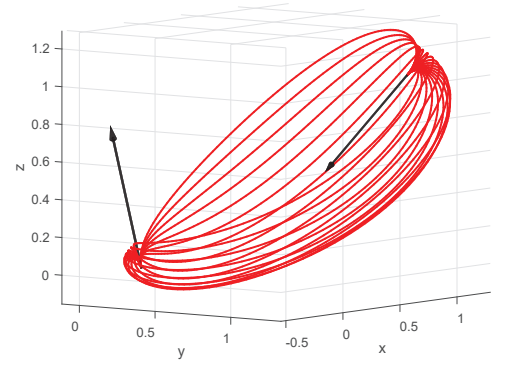

(c) $\alpha=\frac{10}{21} \pi$

Fig. 7 One-parameter families of spatial ATPH Hermite interpolants to the data $\mathbf{p}_{0}=$ $(0,0,0), \mathbf{p}_{5}=(1,1,1), \mathbf{d}_{i}=(-0.8,0.3,1.2), \mathbf{d}_{f}=(0.5,-1.3,-1)$, defined by fixing $\phi_{1}=-\frac{\pi}{2}$, $\phi_{m}=\frac{\pi}{3}$ and varying only $\Delta \phi$ in $[-\pi, \pi]$.

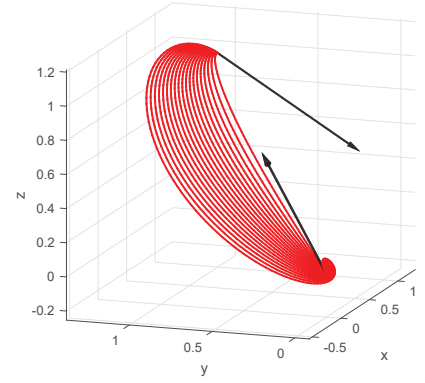

(a) $\Delta \phi=-\frac{3}{5} \pi$

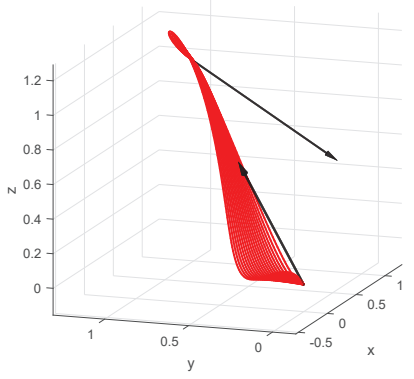

(b) $\Delta \phi=\frac{\pi}{5}$

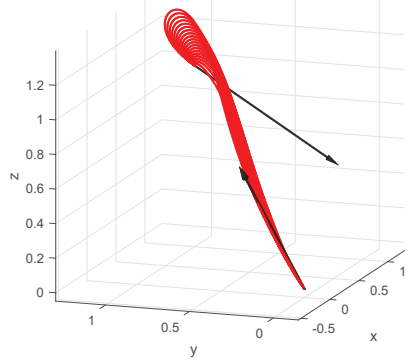

(c) $\Delta \phi=\pi$

Fig. 8 One-parameter families of spatial ATPH Hermite interpolants to the data $\mathbf{p}_{0}=$ $(0,0,0), \mathbf{p}_{5}=(1,1,1), \mathbf{d}_{i}=(-0.8,0.3,1.2), \mathbf{d}_{f}=(0.5,-1.3,-1)$, defined by fixing $\phi_{1}=-\frac{\pi}{2}$, $\phi_{m}=\frac{\pi}{3}$ and varying only $\alpha$ in $\left(0, \frac{2}{3} \pi\right)$.

4.2 A way to fix the angular degrees of freedom $\phi_{0}$ and $\phi_{2}$

Let $\mathbf{r}(t)$ be a spatial ATPH curve. Like in the polynomial case (see [2-4]), we can define the elastic bending energy of $\mathbf{r}(t)$ as

$$
E:=\int_{0}^{\alpha} \kappa^{2}(t)\left|\mathbf{r}^{\prime}(t)\right| d t \quad \text { with } \quad \kappa(t):=\frac{\left|\mathbf{r}^{\prime}(t) \times \mathbf{r}^{\prime \prime}(t)\right|}{\left|\mathbf{r}^{\prime}(t)\right|^{3}}
$$


where, according to (9), $\mathbf{r}^{\prime}(t)=\mathbf{A}(t) \mathbf{i} \mathbf{A}^{*}(t)$ and $\mathbf{r}^{\prime \prime}(t)=\mathbf{A}^{\prime}(t) \mathbf{i} \mathbf{A}^{*}(t)+\mathbf{A}(t) \mathbf{i} \mathbf{A}^{\prime *}(t)$ are both pure vector quaternions (i.e. quaternions of the form $\mathbf{Q}=(0, \mathbf{q})$ with $\left.\mathbf{q}=\operatorname{vect}(\mathbf{Q})=q_{x} \mathbf{i}+q_{y} \mathbf{j}+q_{z} \mathbf{k}\right)$. Since $E$ depends on $\phi_{0}$ and $\phi_{2}$ in a non-linear way, it is very hard to identify the optimal values of the two angular variables which correspond to the global minimum of $E$. However, there is a strong numerical evidence that the angular values $\phi_{0}=\phi_{2}=-\frac{\pi}{2}$ can be used as a default choice to provide ATPH Hermite interpolants of reasonable shape (see Figure 9). In fact, although the choice $\phi_{0}=\phi_{2}=-\frac{\pi}{2}$ does not always correspond to the global minimum of the shape measure $E$, we have observed empirically from many examples that it turns out to be very close to it.

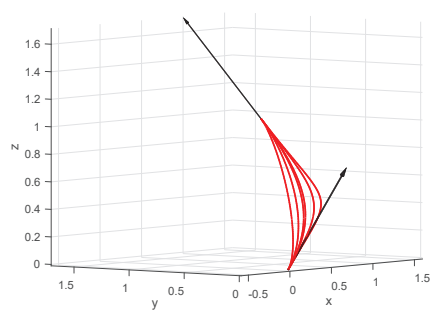

(a) $\phi_{0}=\phi_{2}=-\frac{\pi}{2}$

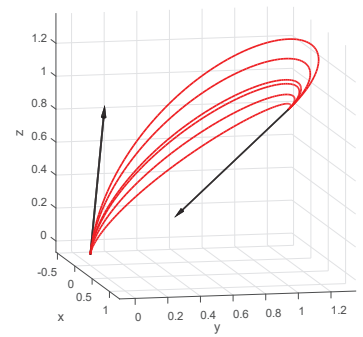

(d) $\phi_{0}=\phi_{2}=-\frac{\pi}{2}$

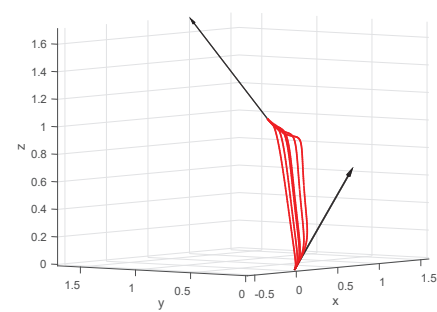

(b) $\phi_{0}=\phi_{2}=0$

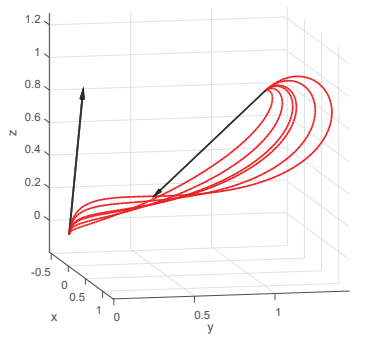

(e) $\phi_{0}=\phi_{2}=0$

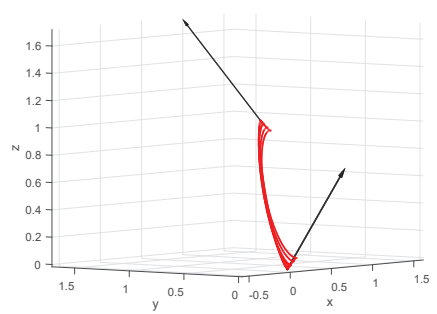

(c) $\phi_{0}=\phi_{2}=\frac{\pi}{2}$

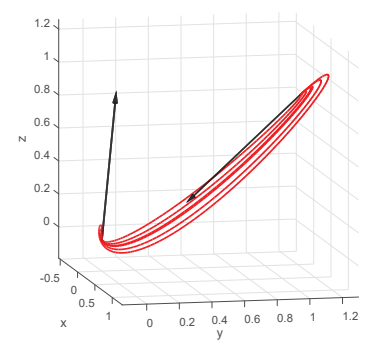

(f) $\phi_{0}=\phi_{2}=\frac{\pi}{2}$

Fig. 9 Comparison between spatial ATPH Hermite interpolants to the data $\mathbf{p}_{0}=(0,0,0)$, $\mathbf{p}_{5}=(1,1,1), \mathbf{d}_{i}=(1,0,1), \mathbf{d}_{f}=(0,1,1)$ (first row) and $\mathbf{p}_{0}=(0,0,0), \mathbf{p}_{5}=(1,1,1)$, $\mathbf{d}_{i}=(-0.8,0.3,1.2), \mathbf{d}_{f}=(0.5,-1.3,-1)$ (second row), obtained by fixing $\phi_{1}=-\frac{\pi}{2}, \alpha \in$ $\{2 / 21 \pi, 4 / 21 \pi, 2 / 7 \pi, 1,10 / 21 \pi, 3 / 5 \pi\}$ and choosing $\phi_{0}, \phi_{2}$ as specified in each subfigure.

\subsection{Benefits of the free parameter $\alpha$}

For given Hermite data and fixed values of $\phi_{0}$ and $\phi_{2}$ (e.g., $\phi_{0}=\phi_{2}=-\frac{\pi}{2}$ ), ATPH interpolants increase the flexibility of $\mathrm{PH}$ interpolants in the sense that they allow the user to:

- use $\alpha$ as a shape parameter (see Figure 10);

- select $\alpha$ to minimize $S$ or $E$ (see Figures 11, 12);

- select $\alpha$ to improve the behaviour of curvature and torsion (see Figure 13). 


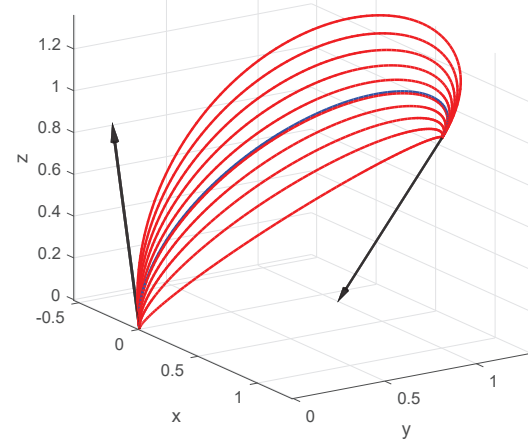

(a) $\mathbf{d}_{i}=(-0.8,0.3,1.2), \mathbf{d}_{f}=(0.5,-1.3,-1)$

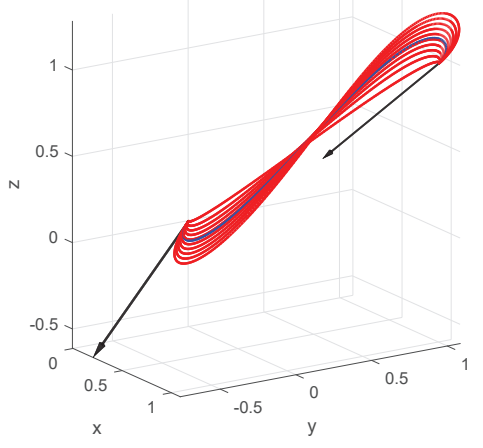

(b) $\mathbf{d}_{i}=(0.4,-1.5,-1.2), \mathbf{d}_{f}=(-1.2,-0.6,-1.2)$

Fig. 10 PH Hermite interpolant (blue) and family of ATPH Hermite interpolants (red) to the end points $\mathbf{p}_{0}=(0,0,0), \mathbf{p}_{5}=(1,1,1)$ and associated end derivatives $\mathbf{d}_{i}, \mathbf{d}_{f}$, obtained by fixing $\phi_{0}=\phi_{1}=\phi_{2}=-\frac{\pi}{2}$. Each curve of the family of ATPH interpolants is identified by a different value of $\alpha \in\left(0, \frac{2}{3} \pi\right)$, precisely, $\alpha_{j}=0.05+\frac{j}{9}\left(\frac{2}{3} \pi-0.1\right), j=0, \ldots, 9$.

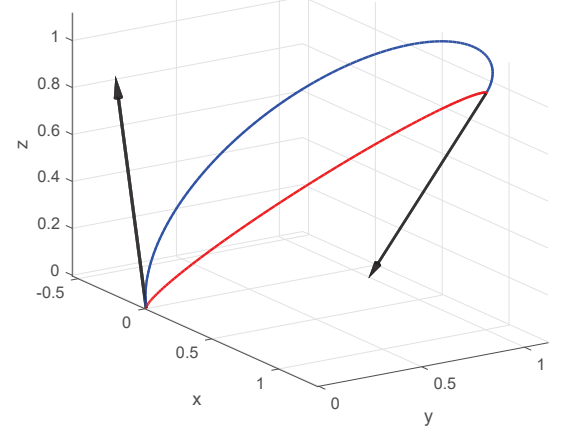

(a) $\alpha=0.05, S=1.7610\left(S^{P H}=2.3104\right)$

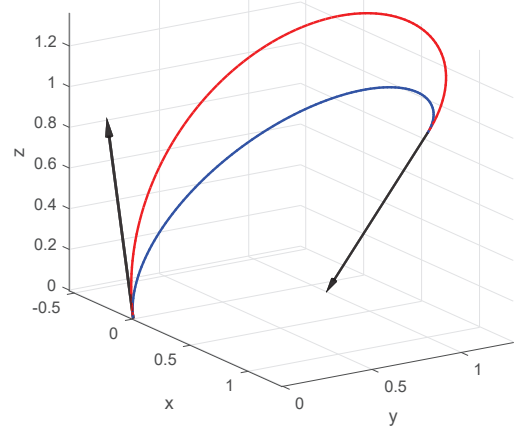

(b) $\alpha=\frac{2}{3} \pi-0.05, E=4.8526\left(E^{P H}=8.8601\right)$

Fig. 11 Blue: PH Hermite interpolant from Figure 10 (a). In (a) the ATPH Hermite interpolant (red) from the family in Figure 10 (a) that minimizes the arc length; in (b) the one which minimizes the elastic bending energy.

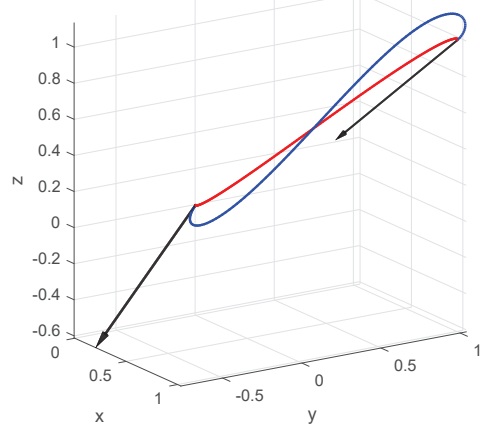

(a) $\alpha=0.05, S=1.7614\left(S^{P H}=2.3181\right)$

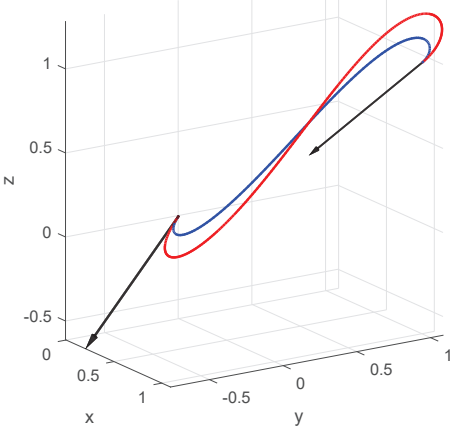

(b) $\alpha=\frac{2}{3} \pi-0.05, E=32.5414\left(E^{P H}=50.4543\right)$

Fig. 12 Blue: PH Hermite interpolant from Figure 10 (b). In (a) the ATPH Hermite interpolant (red) from the family in Figure 10 (b) that minimizes the arc length; in (b) the one which minimizes the elastic bending energy. 


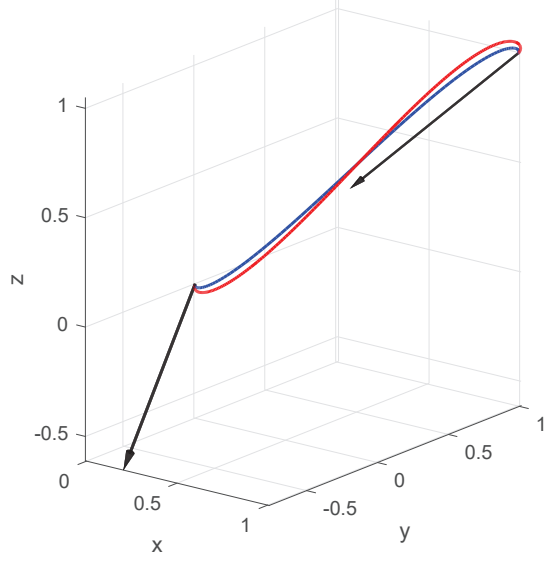

(a)

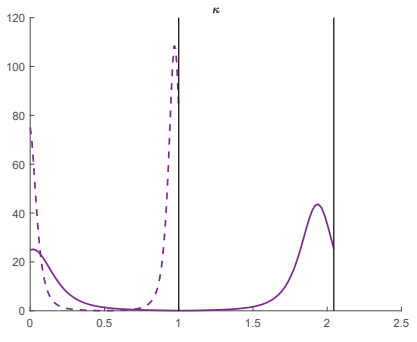

(b)

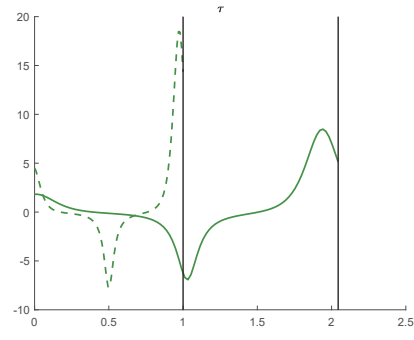

(c)

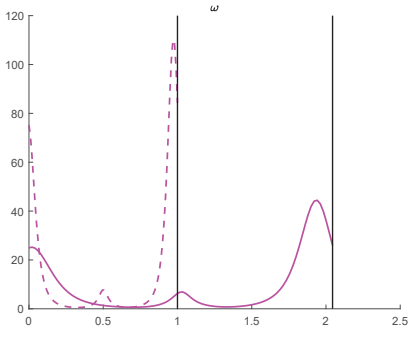

(d)

Fig. 13 In (a) the PH (blue) and the ATPH (red) Hermite interpolant to the data $\mathbf{p}_{0}=$ $(0,0,0), \mathbf{p}_{5}=(1,1,1), \mathbf{d}_{i}=\frac{1}{6}(0.4,-1.5,-1.2), \mathbf{d}_{f}=\frac{1}{6}(-1.2,-0.6,-1.2)$, obtained by fixing $\phi_{0}=\phi_{1}=\phi_{2}=-\frac{\pi}{2}$ and $\alpha=\frac{2}{3} \pi-0.05$. In (b), (c), (d) the behaviour of the curvature $\kappa$, the torsion $\tau$ and the total curvature $\omega:=\sqrt{\kappa^{2}+\tau^{2}}$ of the PH interpolant (dashed lines) and the ATPH interpolant (solid lines).

\section{Reverse engineering of spatial ATPH curves}

Given the control points $\mathbf{p}_{0}, \mathbf{p}_{1}, \ldots, \mathbf{p}_{5}$ of a spatial ATPH curve, the reverse engineering problem consists in determining the three quaternion coefficients $\mathbf{A}_{0}, \mathbf{A}_{1}, \mathbf{A}_{2}$ from the five vector equations

$$
\begin{aligned}
\mathbf{A}_{0} \mathbf{i} \mathbf{A}_{0}^{*} & =\mathbf{d}_{0}, \\
\operatorname{vect}\left(\mathbf{A}_{0} \mathbf{i} \mathbf{A}_{1}^{*}\right) & =\frac{n_{0}}{2\left(n_{0}-6 n_{2}\right)} \mathbf{d}_{1}, \\
\operatorname{vect}\left(\mathbf{A}_{0} \mathbf{i} \mathbf{A}_{2}^{*}\right)+\left(c_{2}+1\right)\left(\mathbf{A}_{1} \mathbf{i} \mathbf{A}_{1}^{*}\right) & =\frac{n_{0}}{4 n_{2}} \mathbf{d}_{2}, \\
\operatorname{vect}\left(\mathbf{A}_{1} \mathbf{i} \mathbf{A}_{2}^{*}\right) & =\frac{n_{0}}{2\left(n_{0}-6 n_{2}\right)} \mathbf{d}_{3}, \\
\mathbf{A}_{2} \mathbf{i} \mathbf{A}_{2}^{*} & =\mathbf{d}_{4},
\end{aligned}
$$

obtained from (10) by setting

$$
\mathbf{d}_{i}:=\frac{16 s_{1}^{4}}{n_{0}}\left(\mathbf{p}_{i+1}-\mathbf{p}_{i}\right), \quad i=0, \ldots, 4
$$

To this end we follow the approach used in [5]. Since we focus our attention on ATPH curves for which $\left|\mathbf{d}_{i}\right| \neq 0, i=0, \ldots, 4$, we are allowed to introduce the 
notation

$$
\boldsymbol{\delta}_{i}:=\frac{\mathbf{d}_{i}}{\left|\mathbf{d}_{i}\right|}, \quad i=0, \ldots, 4 .
$$

If we assume $\boldsymbol{\delta}_{0}, \boldsymbol{\delta}_{4} \neq-\mathbf{i}$, the solution to the first and the fifth equation in (27) can be written in the form

$$
\mathbf{A}_{0}=\sqrt{\left|\mathbf{d}_{0}\right|} \mathbf{m}_{0} \exp \left(\phi_{0} \mathbf{i}\right), \quad \mathbf{A}_{2}=\sqrt{\left|\mathbf{d}_{4}\right|} \mathbf{m}_{4} \exp \left(\phi_{2} \mathbf{i}\right),
$$

where

$$
\mathbf{m}_{0}:=\frac{\mathbf{i}+\boldsymbol{\delta}_{0}}{\left|\mathbf{i}+\boldsymbol{\delta}_{0}\right|}, \quad \mathbf{m}_{4}:=\frac{\mathbf{i}+\boldsymbol{\delta}_{4}}{\left|\mathbf{i}+\boldsymbol{\delta}_{4}\right|} .
$$

On the other hand, from the second equation in (27) we can write

$$
\mathbf{A}_{0} \mathbf{i} \mathbf{A}_{1}^{*}=\frac{n_{0}}{2\left(n_{0}-6 n_{2}\right)}\left(-\xi, \mathbf{d}_{1}\right), \quad \xi \in \mathbb{R},
$$

from which we obtain

$$
\begin{aligned}
\mathbf{i} \mathbf{A}_{1}^{*} & =\frac{n_{0}}{2\left(n_{0}-6 n_{2}\right)\left|\mathbf{A}_{0}\right|^{2}} \mathbf{A}_{0}^{*}\left(-\xi, \mathbf{d}_{1}\right) \\
\mathbf{A}_{1}^{*} & =\frac{n_{0}}{2\left(n_{0}-6 n_{2}\right)\left|\mathbf{A}_{0}\right|^{2}} \mathbf{i}^{*} \mathbf{A}_{0}^{*}\left(-\xi, \mathbf{d}_{1}\right) \\
\mathbf{A}_{1} & =\frac{n_{0}}{2\left(n_{0}-6 n_{2}\right)\left|\mathbf{A}_{0}\right|^{2}}\left(\mathbf{i}^{*} \mathbf{A}_{0}^{*}\left(-\xi, \mathbf{d}_{1}\right)\right)^{*}=\frac{n_{0}}{2\left(n_{0}-6 n_{2}\right)\left|\mathbf{A}_{0}\right|^{2}}\left(-\xi,-\mathbf{d}_{1}\right) \mathbf{A}_{0} \mathbf{i} .
\end{aligned}
$$

Hence, after recalling the expression of $\mathbf{A}_{0}$ in (30), we arrive at

$$
\mathbf{A}_{1}=\frac{n_{0}}{2\left(6 n_{2}-n_{0}\right) \sqrt{\left|\mathbf{d}_{0}\right|}}\left(\xi, \mathbf{d}_{1}\right) \mathbf{m}_{0} \exp \left(\phi_{0} \mathbf{i}\right) \mathbf{i} .
$$

If we now set $\boldsymbol{\delta}_{1}:=\frac{\mathbf{d}_{1}}{\left|\mathbf{d}_{1}\right|}$ and use the expression in (32) to form the product $\mathbf{A}_{1} \mathbf{i} \mathbf{A}_{1}^{*}$, we obtain

$$
\mathbf{A}_{1} \mathbf{i} \mathbf{A}_{1}^{*}=\frac{n_{0}^{2}}{4\left(n_{0}-6 n_{2}\right)^{2}} \frac{\xi^{2} \boldsymbol{\delta}_{0}-2 \xi\left|\mathbf{d}_{1}\right|\left(\boldsymbol{\delta}_{0} \times \boldsymbol{\delta}_{1}\right)+2\left|\mathbf{d}_{1}\right|^{2}\left(\boldsymbol{\delta}_{0} \cdot \boldsymbol{\delta}_{1}\right) \boldsymbol{\delta}_{1}-\left|\mathbf{d}_{1}\right|^{2} \boldsymbol{\delta}_{0}}{\left|\mathbf{d}_{0}\right|} .
$$

Moreover, exploiting (30) we get

$$
\operatorname{vect}\left(\mathbf{A}_{0} \mathbf{i} \mathbf{A}_{2}^{*}\right)=\cos (\Delta \phi) \mathbf{u}+\sin (\Delta \phi) \mathbf{v},
$$

with

$$
\begin{aligned}
\mathbf{u} & :=\sqrt{\left|\mathbf{d}_{0}\right|\left|\mathbf{d}_{4}\right|}\left(\left(\mathbf{m}_{0} \cdot \mathbf{i}\right) \mathbf{m}_{4}+\left(\mathbf{m}_{4} \cdot \mathbf{i}\right) \mathbf{m}_{0}-\left(\mathbf{m}_{0} \cdot \mathbf{m}_{4}\right) \mathbf{i}\right), \\
\mathbf{v} & :=\sqrt{\left|\mathbf{d}_{0}\right|\left|\mathbf{d}_{4}\right|}\left(\mathbf{m}_{4} \times \mathbf{m}_{0}\right),
\end{aligned}
$$

and $\Delta \phi=\phi_{2}-\phi_{0}$. We continue by substituting (33) and (34) into the third equation in (27). This yields the vector condition

$$
\begin{gathered}
\left(c_{2}+1\right) n_{0}^{2} n_{2} \xi^{2} \boldsymbol{\delta}_{0}-2\left(c_{2}+1\right) n_{0}^{2} n_{2} \xi\left|\mathbf{d}_{1}\right|\left(\boldsymbol{\delta}_{0} \times \boldsymbol{\delta}_{1}\right) \\
+4 n_{2}\left(n_{0}-6 n_{2}\right)^{2}\left|\mathbf{d}_{0}\right|(\cos (\Delta \phi) \mathbf{u}+\sin (\Delta \phi) \mathbf{v})= \\
n_{0}\left(n_{0}-6 n_{2}\right)^{2}\left|\mathbf{d}_{0}\right| \mathbf{d}_{2}+\left(c_{2}+1\right) n_{0}^{2} n_{2}\left|\mathbf{d}_{1}\right|^{2} \boldsymbol{\delta}_{0}-2\left(c_{2}+1\right) n_{0}^{2} n_{2}\left|\mathbf{d}_{1}\right|^{2}\left(\boldsymbol{\delta}_{0} \cdot \boldsymbol{\delta}_{1}\right) \boldsymbol{\delta}_{1},
\end{gathered}
$$

which defines an over-determined system of three scalar equations in the two unknowns $\xi$ and $\Delta \phi$. Note that this system is consistent if the given data define a spatial ATPH. In order to solve (36) we proceed as follows. 
First we note that $\left(\mathbf{m}_{0} \cdot \mathbf{i}\right) \mathbf{m}_{0}=\frac{\mathbf{i}+\boldsymbol{\delta}_{0}}{2}$ and $\left(\mathbf{m}_{4} \cdot \mathbf{i}\right) \mathbf{m}_{4}=\frac{\mathbf{i}+\boldsymbol{\delta}_{4}}{2}$, from which we obtain that

$$
\mathbf{u} \times \mathbf{v}=\frac{\left|\mathbf{d}_{0}\right|\left|\mathbf{d}_{4}\right|}{2}\left(\boldsymbol{\delta}_{4}-\boldsymbol{\delta}_{0}\right),
$$

and, under the stated assumptions, $\mathbf{u} \times \mathbf{v} \neq \mathbf{0}$.

Second, we observe that $\mathbf{u} \cdot(\mathbf{u} \times \mathbf{v})=\mathbf{v} \cdot(\mathbf{u} \times \mathbf{v})=0$, which means that by computing the dot product of (36) with $\mathbf{u} \times \mathbf{v}$ we can eliminate the unknown $\Delta \phi$ and obtain a quadratic equation in $\xi$. Precisely, exploiting the fact that

$$
\boldsymbol{\delta}_{0} \cdot(\mathbf{u} \times \mathbf{v})=\frac{\left|\mathbf{d}_{0}\right|\left|\mathbf{d}_{4}\right|}{2}\left(\boldsymbol{\delta}_{0} \cdot \boldsymbol{\delta}_{4}-1\right)
$$

and

$$
\left(\boldsymbol{\delta}_{0} \times \boldsymbol{\delta}_{1}\right) \cdot(\mathbf{u} \times \mathbf{v})=\frac{\left|\mathbf{d}_{0}\right|\left|\mathbf{d}_{4}\right|}{2}\left(\boldsymbol{\delta}_{0} \times \boldsymbol{\delta}_{1}\right) \cdot \boldsymbol{\delta}_{4},
$$

we obtain the quadratic equation

$$
a \xi^{2}+b \xi+c=0
$$

with

$$
\begin{aligned}
a= & \left(c_{2}+1\right) n_{0}^{2} n_{2}\left(\boldsymbol{\delta}_{0} \cdot \boldsymbol{\delta}_{4}-1\right) \\
b= & -2\left(c_{2}+1\right) n_{0}^{2} n_{2}\left|\mathbf{d}_{1}\right|\left(\boldsymbol{\delta}_{0} \times \boldsymbol{\delta}_{1}\right) \cdot \boldsymbol{\delta}_{4}, \\
c= & \left(\boldsymbol{\delta}_{0}-\boldsymbol{\delta}_{4}\right) \cdot\left(n_{0}\left(n_{0}-6 n_{2}\right)^{2}\left|\mathbf{d}_{0}\right| \mathbf{d}_{2}+\left(c_{2}+1\right) n_{0}^{2} n_{2}\left|\mathbf{d}_{1}\right|^{2} \boldsymbol{\delta}_{0}\right. \\
& \left.-2\left(c_{2}+1\right) n_{0}^{2} n_{2}\left|\mathbf{d}_{1}\right|^{2}\left(\boldsymbol{\delta}_{0} \cdot \boldsymbol{\delta}_{1}\right) \boldsymbol{\delta}_{1}\right) .
\end{aligned}
$$

Thus, to guarantee the existence of a solution $\xi \in \mathbb{R}$, the condition $b^{2}-4 a c \geq 0$ must be satisfied by the given data, and this is a non-obvious consequence of the fact that $\mathbf{d}_{0}, \mathbf{d}_{1}, \ldots, \mathbf{d}_{4}$ define a spatial ATPH. Then, for each root $\xi$ of (37), corresponding values of the unknown $\Delta \phi$ can be computed by taking the dot product of (36) with i. Precisely, after introducing the notation

$$
\begin{aligned}
r(\xi):=\mathbf{i} \cdot( & n_{0}\left(n_{0}-6 n_{2}\right)^{2}\left|\mathbf{d}_{0}\right| \mathbf{d}_{2}+\left(c_{2}+1\right) n_{0}^{2} n_{2}\left|\mathbf{d}_{1}\right|^{2} \boldsymbol{\delta}_{0} \\
& -2\left(c_{2}+1\right) n_{0}^{2} n_{2}\left|\mathbf{d}_{1}\right|^{2}\left(\boldsymbol{\delta}_{0} \cdot \boldsymbol{\delta}_{1}\right) \boldsymbol{\delta}_{1} \\
& \left.-\left(c_{2}+1\right) n_{0}^{2} n_{2} \xi^{2} \boldsymbol{\delta}_{0}+2\left(c_{2}+1\right) n_{0}^{2} n_{2} \xi\left|\mathbf{d}_{1}\right|\left(\boldsymbol{\delta}_{0} \times \boldsymbol{\delta}_{1}\right)\right),
\end{aligned}
$$

and

$$
p:=4 n_{2}\left(n_{0}-6 n_{2}\right)^{2}\left|\mathbf{d}_{0}\right|(\mathbf{i} \cdot \mathbf{u}), \quad q:=4 n_{2}\left(n_{0}-6 n_{2}\right)^{2}\left|\mathbf{d}_{0}\right|(\mathbf{i} \cdot \mathbf{v}),
$$

the dot product of (36) with $\mathbf{i}$ yields the equation

$$
p \cos (\Delta \phi)+q \sin (\Delta \phi)=r(\xi) .
$$

Equation (41) can be rewritten as

$$
\cos (\Delta \phi-\psi)=\frac{r(\xi)}{\sqrt{p^{2}+q^{2}}}
$$

with

$$
\cos (\psi)=\frac{p}{\sqrt{p^{2}+q^{2}}} \quad \text { and } \quad \sin (\psi)=\frac{q}{\sqrt{p^{2}+q^{2}}}
$$


There follows that the root $\xi$ must satisfy the condition

$$
|r(\xi)| \leq \sqrt{p^{2}+q^{2}}
$$

in order to obtain from equation (42) the corresponding values $\Delta \phi \in[0,2 \pi]$. Since $\psi \in\left\{\operatorname{acos}\left(\frac{p}{\sqrt{p^{2}+q^{2}}}\right), 2 \pi-\operatorname{acos}\left(\frac{p}{\sqrt{p^{2}+q^{2}}}\right)\right\}$ satisfies (43) and $\Delta \phi \in\left\{\operatorname{acos}\left(\frac{r(\xi)}{\sqrt{p^{2}+q^{2}}}\right)\right.$ $\left.+\psi, 2 \pi-\operatorname{acos}\left(\frac{r(\xi)}{\sqrt{p^{2}+q^{2}}}\right)+\psi\right\}$ satisfies (42), the proposed approach can determine up to eight distinct solutions $(\xi, \Delta \phi)$ of equation (36). For each solution, if (for instance) we assume $\phi_{0}$ to be freely chosen and compute $\phi_{2}=\phi_{0}+\Delta \phi$, the corresponding quaternion coefficients $\mathbf{A}_{0}, \mathbf{A}_{1}, \mathbf{A}_{2}$ may be obtained by equations (30), (32). Note that such coefficients are unique only modulo a common factor $\exp (\phi \mathbf{i})$ for any $\phi$, reflecting the free choice of $\phi_{0}$ (or $\left.\phi_{2}\right)$.

Remark 5 The cases $\boldsymbol{\delta}_{0}, \boldsymbol{\delta}_{4}=-\mathbf{i}$ can be treated in a similar way; thus, for brevity, we discount them.

For the reader's convenience, in the following algorithm we summarize the computational strategy for determining the quaternion coefficients $\mathbf{A}_{0}, \mathbf{A}_{1}, \mathbf{A}_{2}$ from the control points of a spatial ATPH curve.

The reverse engineering algorithm.

Input: the control points $\mathbf{p}_{i}, i=0, \ldots, 5 ; \alpha ; \phi_{0}$.

1. Compute $s_{1}, c_{2}, s_{2}$ by using (2) and then compute $n_{0}, n_{2}$ by using (3).

2. Compute $\mathbf{d}_{0}, \mathbf{d}_{1}, \mathbf{d}_{2}, \mathbf{d}_{4}$ via (28) and then compute $\boldsymbol{\delta}_{0}, \boldsymbol{\delta}_{1}, \boldsymbol{\delta}_{4}$ by using (29).

3. Compute $\mathbf{m}_{0}, \mathbf{m}_{4}$ by using (31).

4. Compute $a, b, c$ by using (38).

5. if $b^{2}-4 a c \geq 0$ do

5.1. Compute $\xi=\frac{-b \pm \sqrt{b^{2}-4 a c}}{2 a}$.

5.2. Compute $r(\xi)$ by using (39), u, v by using (35) and then $p, q$ via (40).

5.3. Compute $\psi=\operatorname{acos}\left(\frac{p}{\sqrt{p^{2}+q^{2}}}\right)$ or $\psi=2 \pi-\operatorname{acos}\left(\frac{p}{\sqrt{p^{2}+q^{2}}}\right)$.

5.4. if $|r(\xi)| \leq \sqrt{p^{2}+q^{2}}$ do 5.4.1 Compute $\Delta \phi=\operatorname{acos}\left(\frac{r(\xi)}{\sqrt{p^{2}+q^{2}}}\right)+\psi$ or $\Delta \phi=2 \pi-a \cos \left(\frac{r(\xi)}{\sqrt{p^{2}+q^{2}}}\right)+\psi$, and set $\phi_{2}=\phi_{0}+\Delta \phi$.

5.4.2 Compute $\mathbf{A}_{j}, j=0,1,2$ by using (30) and (32). end

end

Output: the three quaternion coefficients $\mathbf{A}_{j}, j=0,1,2$.

Remark 6 When the control points of the ATPH curve are constructed by selecting $\phi_{0}=\phi_{2}$, the solutions

$$
\psi=\operatorname{acos}\left(\frac{p}{\sqrt{p^{2}+q^{2}}}\right), \quad \Delta \phi=2 \pi-\operatorname{acos}\left(\frac{r(\xi)}{\sqrt{p^{2}+q^{2}}}\right)+\psi
$$


and

$$
\psi=2 \pi-\operatorname{acos}\left(\frac{p}{\sqrt{p^{2}+q^{2}}}\right), \quad \Delta \phi=\operatorname{acos}\left(\frac{r(\xi)}{\sqrt{p^{2}+q^{2}}}\right)+\psi,
$$

both identify the same three quaternion coefficients $\mathbf{A}_{j}, j=0,1,2$. In fact, $p=$ $r(\xi)$ and the computed $\psi$ is such that $\Delta \phi=2 \pi$.

Conversely, when the control points of the ATPH curve are constructed by selecting $\phi_{0} \neq \phi_{2}$, from our numerical tests we always obtained only one correct solution. The following example is included to illustrate this fact.

Example 1 We select $\alpha=\frac{3}{5} \pi, \phi_{0}=-\frac{2}{5} \pi, \phi_{1}=-\frac{\pi}{2}, \phi_{2}=\frac{9}{20} \pi$ and consider the spatial ATPH curve $\mathbf{r}(t)$ which solves the first-order Hermite interpolation problem

$$
\mathbf{r}(0)=(0,0,0), \mathbf{r}^{\prime}(0)=(-0.8,0.3,1.2), \mathbf{r}(\alpha)=(1,1,1), \mathbf{r}^{\prime}(\alpha)=(0.5,-1.3,-1)
$$

According to the results in Proposition 3, the control points of $\mathbf{r}(t)$ are

$$
\begin{aligned}
& \mathbf{p}_{0}=(0,0,0), \\
& \mathbf{p}_{1}=(-0.363402375465172,0.136275890799440,0.545103563197758), \\
& \mathbf{p}_{2}=(0.446114740676690,0.975310866441397,1.405422626178919), \\
& \mathbf{p}_{3}=(1.896654791102130,0.883305472863396,0.622427428874599), \\
& \mathbf{p}_{4}=(0.772873515334269,1.590528860130905,1.454252969331466), \\
& \mathbf{p}_{5}=(1,1,1) .
\end{aligned}
$$

Applying the reverse-engineering algorithm to obtain from $\mathbf{p}_{0}, \ldots, \mathbf{p}_{5}$ the coefficients $\mathbf{A}_{0}, \mathbf{A}_{1}, \mathbf{A}_{2}$, we find that equation (37) has solutions

$$
\xi_{+}=0.278417655031089 \quad \text { and } \quad \xi_{-}=1.298969111520490
$$

but condition (44) is satisfied by $\xi_{+}$only. Thus four admissible solutions can be worked out which correspond to the choices of $\psi$ in step 5.3 and $\Delta \phi$ in step 5.4.1 of the algorithm. However, for the selected value $\phi_{0}=-\frac{2}{5} \pi$, only one of the four is the correct solution, and it is identified by the choices $\psi=2 \pi-a \cos \left(\frac{p}{\sqrt{p^{2}+q^{2}}}\right)$ and $\Delta \phi=\operatorname{acos}\left(\frac{r\left(\xi_{+}\right)}{\sqrt{p^{2}+q^{2}}}\right)+\psi$. In fact, for these values of $\psi$ and $\Delta \phi$ when recomputing the control points $\mathbf{p}_{1}, \ldots, \mathbf{p}_{5}$ via formula (10), the relative errors

$$
\begin{gathered}
\mathcal{E}_{r}\left(\mathbf{p}_{1}\right)=1.6591 \times 10^{-16}, \quad \mathcal{E}_{r}\left(\mathbf{p}_{2}\right)=1.9859 \times 10^{-16}, \quad \mathcal{E}_{r}\left(\mathbf{p}_{3}\right)=4.4630 \times 10^{-16}, \\
\mathcal{E}_{r}\left(\mathbf{p}_{4}\right)=5.9784 \times 10^{-16}, \quad \mathcal{E}_{r}\left(\mathbf{p}_{5}\right)=7.0509 \times 10^{-16},
\end{gathered}
$$

are found. This is the only case for which the relative errors in $\mathbf{p}_{1}, \ldots, \mathbf{p}_{5}$ have a so small magnitude, so confirming the correctness of this solution only.

By modifying the input data $\phi_{0}$ and $\phi_{2}$ we can also find that condition (44) is satisfied by $\xi_{-}$only, or even by both $\xi_{+}$and $\xi_{-}$. However, in any case, even if eight admissible solutions exist, there is only one choice of $\psi$ and $\Delta \phi$ for which the magnitude of the relative errors in $\mathbf{p}_{1}, \ldots, \mathbf{p}_{5}$ is close to the machine epsilon $2.2204 \times 10^{-16}$. 


\section{Conclusions}

We have presented the new class of spatial ATPH curves. In the same way as general Algebraic-Trigonometric Bézier curves complement general polynomial Bézier curves, the class of spatial ATPH curves provides a beneficial addition to the existing class of spatial PH curves. Moreover, spatial ATPH curves have been shown to inherit the same free parameter that characterizes planar ATPH curves and its benefits in free-form design applications have been illustrated.

\section{References}

1. Carnicer, J.M., Peña, J.M.: Totally positive bases for shape preserving curve design and optimality of B-splines. Comput. Aided Geom. Design 11, 635-656 (1994)

2. Farouki, R.T.: Pythagorean-hodograph curves: algebra and geometry inseparable. Springer, Berlin, 2008.

3. Farouki, R.T., al-Kandari, M., Sakkalis, T.: Hermite interpolation by rotation-invariant spatial Pythagorean-hodograph curves. Adv. Comput. Math. 17, 369-383 (2002)

4. Farouki, R.T., Giannelli, C., Manni, C., Sestini, A.: Identification of spatial PH quintic Hermite interpolants with near-optimal shape measures. Comput. Aided Geom. Design 25(45), 274-297 (2008)

5. Farouki, R.T., Giannelli, C., Sestini, A.: Identification and reverse engineering of Pythagorean-hodograph curves. Comput. Aided Geom. Design 34, 21-36 (2015)

6. Farouki, R.T., Sakkalis, T.: Pythagorean-hodograph space curves. Adv. Comput. Math. 2, 41-66 (1994)

7. Kosinka, J., Lávicka, M.: Pythagorean Hodograph curves: A survey of recent advances. J. Geom. Graph. 18(1), 23-43 (2014)

8. Kozak, J., Krajnc, M., Rogina, M., Vitrih, V.: Pythagorean-hodograph cycloidal curves. J. Numer. Math. 23(4), 345-360 (2015)

9. Mainar, E., Peña, J.M.: Corner cutting algorithms associated with optimal shape preserving representations. Comput. Aided Geom. Des. 16, 883-906 (1999)

10. Mainar, E., Peña, J.M.: Optimal bases for a class of mixed spaces and their associated spline spaces. Comput. Math. Appl. 59, 1509-1523 (2010)

11. Mainar, E., Peña, J.M., Sánchez-Reyes, J.: Shape preserving alternatives to the rational Bézier model. Comput. Aided Geom. Des. 18, 37-60 (2001)

12. González, C., Albrecht, G., Paluszny, M. and Lentini, M.: Design of $C^{2}$ algebraictrigonometric pythagorean-hodograph splines with shape parameters. Comp. Appl. Math. (2016), in press, DOI: 10.1007/s40314-016-0404-y

13. Romani, L., Saini, L., Albrecht, G.: Algebraic-Trigonometric Pythagorean-Hodograph curves and their use for Hermite interpolation. Adv. Comput. Math. 40(56), 977-1010 (2014) 